\title{
NUMERICAL SIMULATIONS FOR NODAL DOMAINS AND SPECTRAL MINIMAL PARTITIONS
}

\author{
Virginie Bonnaillie-NoËL ${ }^{1}$, Bernard HelfFer $^{2}$ ANd Gregory Vial ${ }^{1}$
}

\begin{abstract}
We recall here some theoretical results of Helffer et al. [Ann. Inst. H. Poincaré Anal. Non Linéaire (2007) doi:10.1016/j.anihpc.2007.07.004] about minimal partitions and propose numerical computations to check some of their published or unpublished conjectures and exhibit new ones.
\end{abstract}

Mathematics Subject Classification. 35P05, 65N25, 65N30, 49Q10.

Received September 14, 2007. Revised May 19, 2008 and September 22, 2008.

Published online December 19, 2008.

\section{INTRODUCTION}

We are interested in the properties of the 'minimal' $k$-partitions of an open set $\Omega$ by $k$ disjoint open sets $D_{i}(i=1, \ldots, k)$ in $\Omega$. These partitions are minimal in the sense that they minimize the maximum over $i=1, \ldots, k$ of the lowest eigenvalues of the Dirichlet realization of the Laplacian in $D_{i}$. The corresponding minimum is denoted by $\mathfrak{L}_{k}(\Omega)$. Such problems naturally appear in Biomathematics. In particular, we would like to determine in which cases this minimal partition is actually the family of the nodal domains of a given eigenfunction of the two-dimensional Dirichlet Laplacian in $\Omega$. In the case of 2-partitions, the answer is very simple because a variational characterization of the second eigenvalue of the Dirichlet Laplacian in $\Omega$ shows that a minimal 2-partition is always a nodal partition corresponding to the second eigenvalue. So the interesting questions start with $k=3$. Although general properties of these minimal partitions have been proved in [16] by Helffer et al. (see also [12] for a survey - in French), there are very few theoretical results (except for thin rectangles) for obtaining an explicit determination of minimal partitions. This is already the case for 3 -partitions and for simple cases like the square or the disk. For these reasons, it is particularly useful to mix some theoretical remarks of [16] and still unpublished results of [15] with efficient numerical computations.

The aim of this paper is twofold. First, we discuss when a minimal partition of $\Omega$ corresponds to the nodal domains of an eigenfunction of the Dirichlet Laplacian in $\Omega$ and propose candidates for minimal 3-partitions. Secondly, we investigate the asymptotic behavior of $\mathfrak{L}_{k}(\Omega) / k$ as $k \rightarrow+\infty$ in relation with plane tilings.

The paper is organized as follows: in Section 2, we recall the definition and main known results about minimal partitions, necessary conditions are given which conduct the numerical strategy developed in Section 3. We restrict ourselves to symmetric partitions for which we give an exhaustive classification. For some simple

\footnotetext{
Keywords and phrases. Eigenmodes of Laplace operator, minimal partitions, nodal domains, finite element method.

1 IRMAR, ENS Cachan Bretagne, CNRS, UEB, avenue Robert Schuman, 35170 Bruz, France.

Virginie.Bonnaillie@bretagne.ens-cachan.fr; Gregory.Vial@bretagne.ens-cachan.fr

${ }^{2}$ Laboratoire de Mathématiques, Bâtiment 425, Université Paris-Sud et CNRS, 91405 Orsay Cedex, France.

Bernard.Helffer@math.u-psud.fr
} 
shapes (square, disk, union of three hexagons), new candidates for the minimal 3-partition are exhibited solving numerically a mixed problem in the half-domain. The last section is devoted to the asymptotic behavior as $k \rightarrow+\infty$ of $\mathfrak{L}_{k}(\Omega) / k$ for which we give bounds. Numerical simulations are carried out to test the conjecture $\mathfrak{L}_{k}(\Omega) / k \rightarrow \lambda_{1}\left(\mathrm{Hexa}_{1}\right)$.

All the numerical results presented in this paper have been obtained with the Finite Element Library Mélina, see [20]. The computation reduces to the determination of eigenfunctions of the Dirichlet Laplacian in polygonal domains. We make use of a standard Lagrangian nodal elements coupled with a subspace iteration method. Though classical, these methods have to be applied with a special care in order to obtain high accuracy. Indeed, the determination of nodal lines requires very precise approximation of the eigenfunctions (especially for large ranks). The choice of the package Mélina is motivated by the implementation of high order elements: quadrangles of degree up to 20 and triangles up to degree 6. Besides some corner singularities may appear suggesting a local mesh refinement. It turns out that our computations on a quasi-uniform mesh are accurate enough to catch these possible singularities.

\section{Minimal partition: Survey of KnOWn Results}

\subsection{Main definitions}

Let $\Omega$ be a bounded and regular (i.e. piecewise $C^{1, \alpha}$ for some $\alpha>0$ ) connected domain in $\mathbb{R}^{2}$. The eigenvalues of the Dirichlet realization of the Laplacian $-\Delta$ in $\Omega$ are denoted by

$$
\lambda_{1}<\lambda_{2} \leq \lambda_{3} \leq \ldots \leq \lambda_{n} \ldots
$$

We choose the associated eigenfunctions $u_{n}\left(n \in \mathbb{N}^{*}\right)$ to form an orthonormal basis for $L^{2}(\Omega)$.

Let $k \geq 1$ be an integer. A $k$-weak-partition (in short ' $k$-partition' or simply 'partition') of $\Omega$ is a family $\mathcal{D}=\left(D_{i}\right)_{i=1}^{\bar{k}}$ of mutually disjoint sets such that

$$
\cup_{i=1}^{k} D_{i} \subset \Omega
$$

The weak partition is called open, resp. connected, regular, if the $D_{i}$ are open, resp. connected, regular (i.e. piecewise $C^{1, \alpha}$ for some $\alpha>0$ and with the interior cone condition) sets of $\Omega$. The partition is called strong if

$$
\operatorname{Int}\left(\overline{\cup_{i} D_{i}}\right) \backslash \partial \Omega=\Omega .
$$

The set of open connected weak $k$-partitions of $\Omega$ is denoted by $\mathcal{D}_{k}(\Omega)$.

For $\mathcal{D} \in \mathcal{D}_{k}(\Omega)$, we define

$$
\Lambda(\mathcal{D})=\max \left\{\lambda_{1}\left(D_{i}\right), i=1, \ldots, k\right\}
$$

where $\lambda_{1}\left(D_{i}\right)$ denotes the first eigenvalue ${ }^{1}$ of the Dirichlet realization of the Laplacian in $D_{i}$.

For any integer $k \geq 1$, we define

$$
\mathfrak{L}_{k}(\Omega)=\inf \left\{\Lambda(\mathcal{D}), \mathcal{D} \in \mathcal{D}_{k}\right\}
$$

A weak $k$-partition $\mathcal{D} \in \mathcal{D}_{k}(\Omega)$ such that $\Lambda(\mathcal{D})=\mathfrak{L}_{k}(\Omega)$ is called minimal $k$-partition of $\Omega$.

Let $u \in C_{0}^{0}(\bar{\Omega})$. The nodal domains of $u$ (whose number is denoted by $\mu(u)$ ) are the components of $\Omega \backslash N(u$ ) where

$$
N(u)=\overline{\{x \in \Omega \mid u(x)=0\}} .
$$

When $u$ is an eigenfunction of the Laplacian, $N(u)$ is a $C^{\infty}$ curve, except at some isolated critical points of $\Omega$. The nodal domains of $u$ form a partition of $\Omega$ which is called nodal partition. In a sufficiently small neighborhood of one critical point $x_{c} \in \Omega, N(u)$ is a union of an even number of half-curves meeting at $x_{c}$, with tangents crossing with equal angles. At the points $x_{b}$ of $N(u) \cap \partial \Omega$, we have the analogous property that $N(u)$ is locally a union of half-curves ending at $x_{b}$ with equal angle with the boundary. This will be referred to as 'equal

\footnotetext{
${ }^{1}$ Note that this can be defined in some extended sense for any open set.
} 
angle meeting property' (see [1-3]). This property is proved by approximating $u$ locally by an harmonic polynomial function and analyzing the zeros of this new function.

\subsection{Minimal partitions and eigenmodes}

We briefly recall in this section the main results obtained in [16] and emphasize specific results which motivate the strategy used for the numerical computations.

The first result obtained in [16] is that

Theorem 2.1. A minimal partition has always a strong representative ${ }^{2}$ which is regular.

So the subleties about weak and strong partitions do not play a role in our numerical computations as soon as we are concerned with minimal partitions. The existence of such minimal partitions was previously obtained in [8-10] (see also [7] and an earlier contribution of [5] giving a weaker result).

It has been shown in [16] that the minimal partitions share with the nodal sets many properties. In particular they satisfy the equal angle meeting property but note that the number of half-curves meeting at a critical point may now be odd.

We recall that minimal 2-partitions of $\Omega$ are actually nodal partitions associated with some eigenfunction in the eigenspace corresponding to the second eigenvalue $\lambda_{2}(\Omega)$ of the Dirichlet realization of $-\Delta$ in $\Omega$ :

$$
\mathfrak{L}_{2}(\Omega)=\lambda_{2}(\Omega),
$$

which can be understood as a kind of variational characterization of the second eigenvalue. Hence, it is natural to discuss if this result extends for $k \geq 3$ : does any minimal partition correspond to a nodal partition?

Two subdomains $D_{i}, D_{j}$ are said to be neighbors $\left(D_{i} \sim D_{j}\right)$ if $\operatorname{Int}\left(\overline{D_{i} \cup D_{j}}\right) \backslash \partial \Omega$ is connected. To each $\mathcal{D}$ corresponds a graph $G(\mathcal{D})$ obtained by associating a vertex to each $D_{i}$ and an edge to each pair $D_{i} \sim D_{j}$. This graph is undirected without multiple edges or loops. It is said bipartite if it can be colored by two colors (two neighbours having distinct colors). It is well known that nodal partitions are bipartite. The following converse is deeper (see [14], [8-10] and [16]):

Theorem 2.2. If the graph of the minimal partition is bipartite, this is the nodal partition of an eigenfunction corresponding to $\mathfrak{L}_{k}(\Omega)$.

Theorem 2.3 (Courant's nodal theorem). Let $k \geq 1, \lambda_{k}(\Omega)$ be the $k$-th eigenvalue and $u$ any real eigenfunction of $-\Delta$ on $\Omega$ (so that $-\Delta u=\lambda_{k} u$ ). Then the number of nodal domains $\mu(u)$ of u satisfies

$$
\mu(u) \leq k .
$$

If $\mu(u)=k$, we say that $u$ is Courant-sharp.

For any integer $k \geq 1$, we denote by $L_{k}$ the smallest eigenvalue whose eigenspace contains an eigenfunction with $k$ nodal domains. In general, we have the first comparison (for the proof of Thm. 2.4 and the following ones, see [16])

Theorem 2.4. Let $\Omega$ be a regular open set in $\mathbb{R}^{2}$, then, for any $k \in \mathbb{N}^{*}$, we have

$$
\lambda_{k}(\Omega) \leq \mathfrak{L}_{k}(\Omega) \leq L_{k}(\Omega) .
$$

The classical Pleijel theorem says that $\lambda_{k}(\Omega)<L_{k}(\Omega)$ for $k$ large. In other words, an eigenfunction cannot be Courant-sharp for $k$ large.

An improved version of Pleijel's theorem (which implies this theorem) says:

\footnotetext{
${ }^{2}$ Modulo sets of capacity 0.
} 
Theorem 2.5 (Pleijel's theorem for minimal partitions). Let $\Omega$ be a regular open set in $\mathbb{R}^{2}$, then there exists $k_{0}$ such that, for $k \geq k_{0}$, we have

$$
\lambda_{k}(\Omega)<\mathfrak{L}_{k}(\Omega) .
$$

This in particularly says that a minimal partition cannot be nodal for $k$ large. The proof of this result (see [16]) is based on the Faber-Krahn inequality together with the Weyl formula.

It is interesting to determine the equality cases. It is fulfilled for $k=1$ and $k=2$, it is a mere consequence of the sign properties of the first two eigenfunctions. So the interesting question starts with $k=3$. It is not too difficult to see that for the square and the disk, the two inequalities are strict and that on the contrary, we have again equality for $k=4$.

Theorem 2.6. We assume that $\Omega$ is regular. If $\mathfrak{L}_{k}(\Omega)=L_{k}(\Omega)$ or if $\mathfrak{L}_{k}(\Omega)=\lambda_{k}(\Omega)$, then

$$
\lambda_{k}(\Omega)=\mathfrak{L}_{k}(\Omega)=L_{k}(\Omega) .
$$

Furthermore, there exists in this case an eigenfunction $u_{k}$ in the eigenspace associated to $\lambda_{k}$ such that $\mu\left(u_{k}\right)=k$ (i.e. $u_{k}$ is Courant-sharp).

In other words, the only case when a minimal partition is a nodal partition is the case when this nodal partition corresponds to a Courant-sharp case.

Let us close this short presentation of minimal partitions by a monotonicity property:

Proposition 2.7. If $\Omega \subset \widehat{\Omega}$, then

$$
\mathfrak{L}_{k}(\widehat{\Omega}) \leq \mathfrak{L}_{k}(\Omega) .
$$

Here it is important to notice that a strong partition of $\Omega$ is a weak partition of $\widehat{\Omega}$.

\subsection{Necessary conditions for minimal partitions}

It is not straightforward to find a good algorithm for determining numerically ${ }^{3}$ minimal partitions. So it is interesting to look for necessary conditions which are easier to analyze.

A first necessary condition is that:

Proposition 2.8. Let $k \geq 3$ and $\mathcal{D}=\left(D_{1}, \ldots, D_{k}\right)$ a minimal $k$-partition of $\Omega$. Then, for any pair of neighbours $D_{i} \sim D_{j}, \mathfrak{L}_{k}(\Omega)$ should be the second eigenvalue of the Dirichlet Laplacian of ${ }^{4}$

$$
D_{i j}=\operatorname{Int}\left(\overline{D_{i}} \cup \overline{D_{j}}\right)
$$

$D_{i}$ and $D_{j}$ being the nodal sets of some corresponding eigenfunction.

This is actually a particular case of the more general result which concerns any connected subpartition:

Theorem 2.9. Let $\mathcal{D}$ be a minimal k-partition of $\Omega$ relative to $\mathfrak{L}_{k}(\Omega)$. Let $\Omega^{\prime} \subset \Omega$ be connected and $\mathcal{D}^{\prime} \subset \mathcal{D}$ be any subpartition of $\mathcal{D}$ into $k^{\prime}$ elements $\left(1 \leq k^{\prime}<k\right)$ such that

$$
\overline{\Omega^{\prime}}=\cup\left\{\overline{D_{i}}, D_{i} \in \mathcal{D}^{\prime}\right\} .
$$

Then $\mathfrak{L}_{k}(\Omega)=\mathfrak{L}_{k^{\prime}}\left(\Omega^{\prime}\right)$ and $\mathfrak{L}_{k^{\prime}}\left(\Omega^{\prime}\right)$ is uniquely achieved.

So any subpartition should be minimal and, implementing previous other results, we also see that

\footnotetext{
${ }^{3}$ See however what can be done by using the associated variational problem appearing in the proof of Conti-Terracini-Verzini, or evolutionary algorithms, see [18]. Other numerical approaches are also proposed in [6,11].

${ }^{4}$ Here we recall that, for a given set $U$ in $\mathbb{R}^{2}, \operatorname{Int} U$ denotes the interior of $U$, i.e. the largest open set contained in $U$.
} 

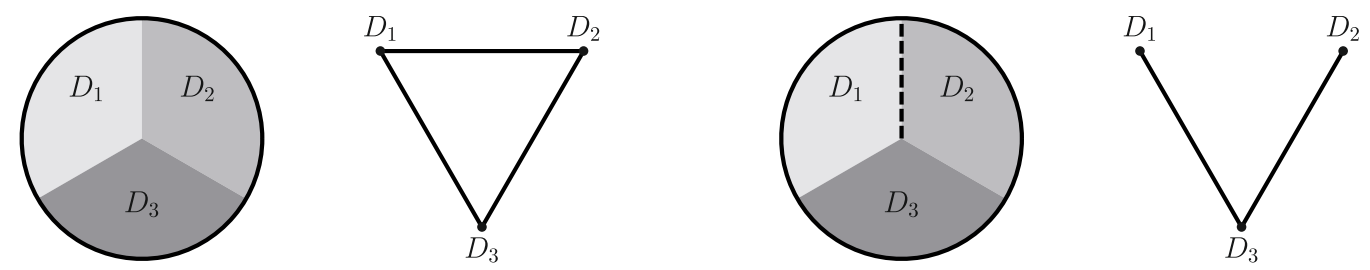

FigURE 1. Extracted connected open set (left: $\Omega$, right: $\widetilde{\Omega}$ ) and corresponding graphs.

Corollary 2.10. With the assumptions of Theorem 2.9, any subpartition with corresponding bipartite subgraph is a nodal partition. Moreover, it then corresponds to a Courant-sharp situation:

$$
\mathfrak{L}_{k^{\prime}}\left(\Omega^{\prime}\right)=\lambda_{k^{\prime}}\left(\Omega^{\prime}\right) .
$$

In the paper [16], there is also implicitly another interesting necessary condition extending the previous theorem. Starting from a minimal regular $k$-partition $\mathcal{D}$ of a domain $\Omega$, we can construct in $\Omega$ a connected domain $\widetilde{\Omega}$ such that $\mathcal{D}$ becomes a minimal bipartite $k$-partition of $\widetilde{\Omega}$. It is achieved by removing from $\Omega$ a union of a finite number of regular arcs corresponding to pieces of boundaries between two neighbours of the partition. Note that this construction can be done in many ways. For an example, see Figures 1 and 24. We say in this case that $\widetilde{\Omega}$ is an extracted open set associated to $\mathcal{D}$.

As a second corollary, we obtain

Corollary 2.11. If $\mathcal{D}$ is a minimal regular $k$-partition, then for any extracted connected open set $\widetilde{\Omega}$ associated with $\mathcal{D}$, we have

$$
\lambda_{k}(\widetilde{\Omega})=\mathfrak{L}_{k}(\widetilde{\Omega}) .
$$

This last criterion will be analyzed below for union of triangles, squares and hexagons as a test of minimality. The numerical computations will show a quite different behavior, which in the two first cases was expected by the theory as we will see in Section 4.3.

\section{Minimal Partitions AND SyMmetries}

If the domain has symmetries, it is natural to wonder whether the symmetry properties are reflected in the properties of the minimal partitions. It is obviously the case for 2-partitions (since they are nodal). In Section 3.1, we give a classification of all minimal possible bipartite 3-partitions (which may be associated to the nodal set of the third eigenfunction) and non bipartite ones (which divide into three configurations). Computations are made in the latter case to provide candidates for minimal 3-partitions, using symmetry to reduce the analysis to the determination of the nodal sets of the second eigenfunction of a mixed problem (see Sects. 3.2 and 3.3).

\subsection{Topological configurations}

We assume that the domain $\Omega$ has a symmetry $\sigma$ with respect to some axis $\left(\right.$ i.e. $\sigma(\Omega)=\Omega$ with $\sigma^{2}=I d$ ) and that, instead of minimizing over all the partitions, we only minimize over 'symmetric' partitions, i.e. partitions which satisfy either

or (after a possible relabeling)

$$
\sigma\left(D_{i}\right)=D_{i}, \text { for } i=1, \ldots, 3
$$

$$
\sigma\left(D_{1}\right)=D_{2}, \sigma\left(D_{3}\right)=D_{3} .
$$

The proof giving the existence of a minimal partition goes through in the symmetric case (i.e. when we minimize over 3-partitions satisfying (3.2)) and so it is natural to look for a symmetric minimizer. 


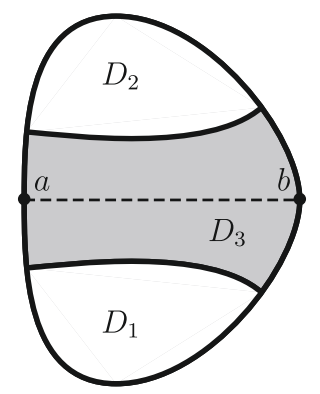

Figure 2. The 3-partition has no critical point.
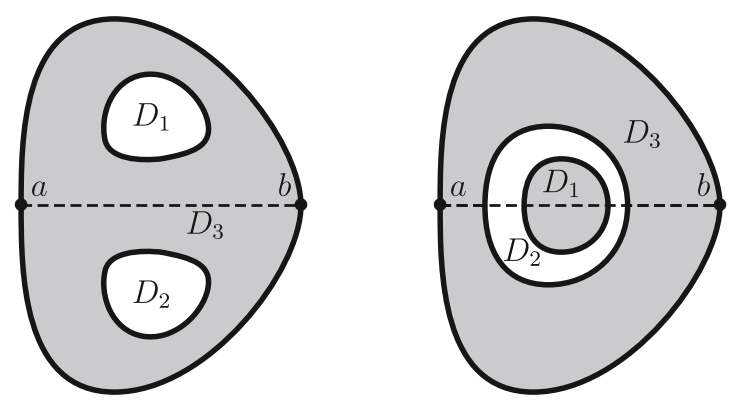

FiguRE 3. The 3-partition has two disjoint circles.

Let us add two notions attached to a strong regular open partition. We call critical point a point which lies at the intersection of the boundaries of at least three open sets of the partition. We call boundary point a point at the intersection of the boundary of $\Omega$ with at least the boundaries of two open sets of the partition. In the example of Figure $6, x_{0}$ is a critical point and $a$ is a boundary point.

It follows from the Euler formula (see [15]) that these minimizers can be classified: we distinguish between bipartite and non bipartite 3-partitions and for each case, we give an exhaustive list of possible configurations.

\section{Bipartite 3-partition}

A first possibility is that the minimal 3-partition is bipartite (hence a nodal domain). The most natural case is illustrated in Figure 2. In the situation of Figure 2, the minimal 3-partition corresponds to the third nodal partition of $\Omega$. This case effectively occurs for a thin vertical rectangle. For general $\Omega$ 's, numerics can help to determine if the third eigenfunction is Courant-sharp. Apart from this case, there are many other possibilities which cannot be excluded a priori:

- 3-partitions with no critical point: this structure brings into play two disjoint "circles" (this provides two configurations: the first one with disjoint 'disks' corresponding to the circles, the second one with one 'circle' inside the 'disk' of the other, see Fig. 3);

- 3-partitions with two boundary points (see Fig. 4) which can be described with one 'circle' and one 'line' joining two points of the boundary;

- 3-partitions with one double point (see Fig. 5) which can be gathered in topological sets: a closed line with a double point and with $D_{1}$ and $D_{2}$ on both sides or one inside the other, or a line joining two points of the boundary but with a double point.

\footnotetext{
${ }^{5} \mathrm{~A}$ 'circle' is simply a closed curve without critical point.
} 


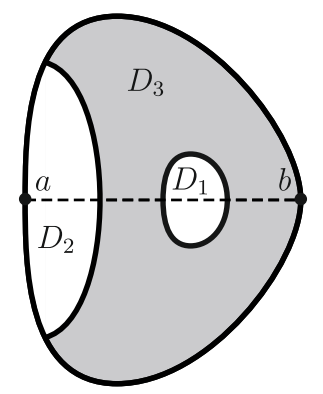

FIGURE 4. The 3-partition has one circle and one line.
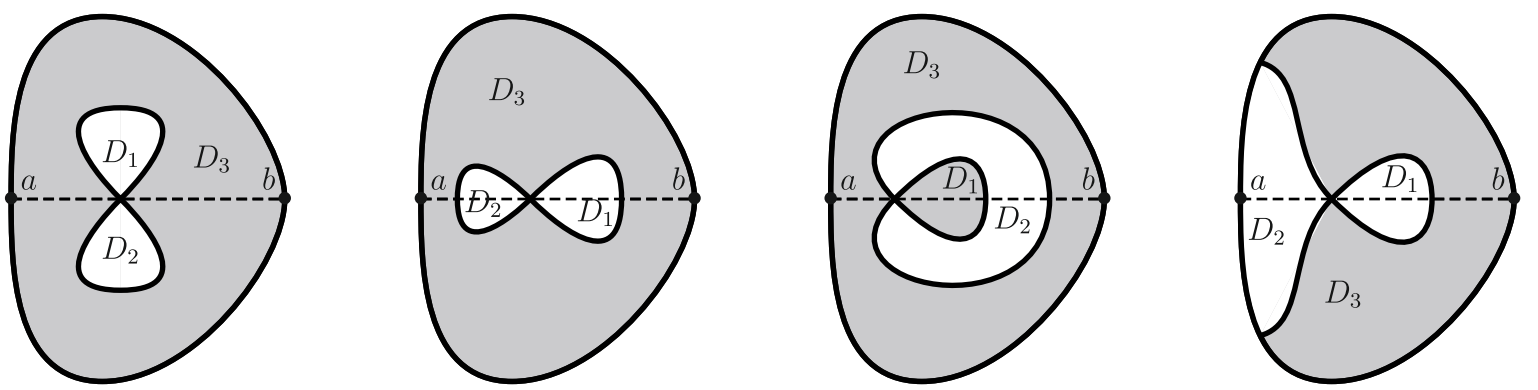

Figure 5. The 3-partition has one double point.

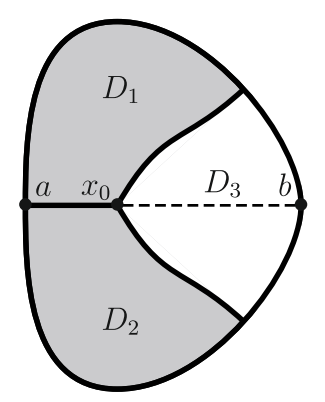

Figure 6 . The 3 -partition has one critical point.

Any of the above bipartite 3-partitions can be obtained numerically, if it exists. All we need is to compute the third eigenfunction and to determine its nodal domains. If the third eigenfunction is Courant-sharp, it provides such a bipartite 3 -partition.

\section{Non bipartite 3-partition}

The second possibility is the case when the minimal partition is not bipartite. We can only have one of the three following structures whose topology is illustrated in Figures 6-8.

(a) The 3-partition has one critical point, which is necessarily on the symmetry axis (cf. Fig. 6).

(b) The 3-partition has two critical points and no boundary point (cf. Fig. 7). 


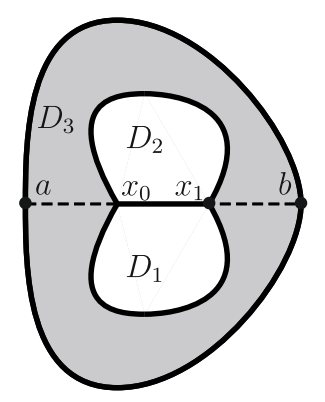

Figure 7. The 3-partition has two critical points and no boundary point.

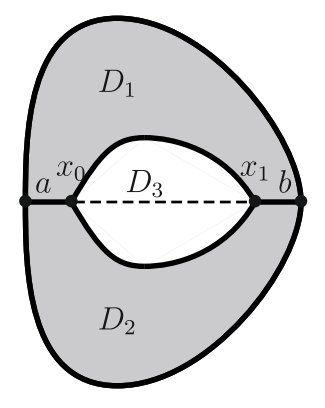

FIGURE 8 . The 3 -partition has two critical points and two boundary points.

(c) The 3-partition has two critical points and two boundary points. Moreover $\partial D_{1} \cap \partial D_{2}$ consists of two segments on the symmetry axis, each one joining one boundary point to one critical point (cf. Fig. 8).

We now assume that the minimal 3-partition is not bipartite. We want to investigate numerically if one of the previous configurations provides a good candidate for being a minimal symmetric 3-partition. For this purpose, we deal with each case separately.

\subsection{Non bipartite 3-partition with one critical point}

We focus here on case (a). Let us define the notations illustrated in Figure 6. We assume that the symmetry axis is $y=0$ in $\mathbb{R}_{x, y}^{2}$ and we denote by $x_{0}$ the critical point. We assume that $\Omega$ is convex to simplify the discussion and denote by $(a, b)$ the segment $\Omega \cap\{y=0\}$. Without loss of generality, we may assume that $\partial D_{1} \cap \partial D_{2}$ is the segment $\left[a, x_{0}\right]$ and that $\partial D_{1} \cap \partial D_{3}$ consists of a line joining (without any selfintersection) $x_{0}$ to $\partial \Omega \cap\{y>0\}$.

If $\mathcal{D}=\left(D_{1}, D_{2}, D_{3}\right)$ is a minimal partition of type a, then $\left(D_{1}, D_{3}\right)$ is a minimal 2-partition and hence the nodal partition associated with the second eigenvalue of $D_{13}=\operatorname{Int}\left(\overline{D_{1}} \cup \overline{D_{3}}\right)$ (cf. Cor. 2.10). Restricting the corresponding eigenfunction to $\Omega^{+}=\Omega \cap\{y>0\}$, we obtain an eigenfunction $\varphi$ of the mixed Dirichlet-Neumann problem illustrated in Figure 9:

$$
-\Delta \varphi=\lambda \varphi \text { in } \Omega^{+}, \quad \partial_{\mathbf{n}} \varphi=0 \text { on }\left[x_{0}, b\right] \quad \text { and } \quad \varphi=0 \text { elsewhere on the boundary. }
$$

Note that $\varphi$ is not necessarily the second eigenfunction.

Nevertheless we focus on the second eigenmode $\left(\lambda_{2}\left(x_{0}\right), \varphi_{x_{0}}\right)$ of the mixed problem (see Rem. 3.1 for the following ones). It is not clear whether $\varphi_{x_{0}}$ has a closed nodal line or not (Melas [21] only proved the non existence in the convex case with Dirichlet conditions). The following remarks settle our strategy:

- The mapping $x_{0} \mapsto \lambda_{2}\left(x_{0}\right)$ is an increasing function. 


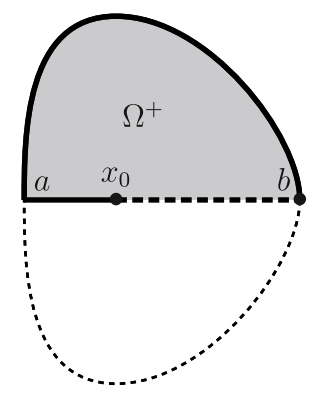

Figure 9. Dirichlet-Neumann problem.

- After symmetrization, a nodal line joining a point of the segment $\left[x_{0}, b\right]$ to a point of $\partial \Omega^{+} \cap\{y>0\}$ only leads to a 2-partition of $\Omega$.

- If the nodal line starts from $\xi \in\left(a, x_{0}\right)$ and reaches the boundary $\partial \Omega^{+} \cap\{y>0\}$, the 3-partition obtained after symmetrization may be improved by removing the segment $\left(\xi, x_{0}\right)$.

Altogether, moving $x_{0}$ along the segment $[a, b]$, we expect the second eigenfunction to have a nodal line joining $x_{0}$ to the boundary $\partial \Omega \cap\{y>0\}$. The smallest $x_{0}$ corresponding to this configuration (if it exists) is denoted by $x_{0}^{*}$. The eigenvalue $\lambda_{2}\left(x_{0}^{*}\right)$ provides an upper bound of $\mathfrak{L}_{3}(\Omega)$ and the nodal domains of the associated eigenfunction $\varphi_{x_{0}^{*}}$ give a possible candidate for the minimal 3-partition of $\Omega$.

From the equal angle meeting property, we know that the nodal line joining $x_{0}^{*}$ to the boundary $\partial \Omega \cap\{y>0\}$ and the segment $\left[a, x_{0}^{*}\right]$ meet at $x_{0}^{*}$ with an angle of $2 \pi / 3$. If $x_{0} \neq x_{0}^{*}$, then the nodal line is orthogonal to $[a, b]$ since, after symmetrization, the point $x_{0}$ is the intersection point of two half-curves if $a<x_{0}<x_{0}^{*}$ (the nodal line joining $x_{0}$ to the boundary $\partial \Omega \cap\{y>0\}$ and its symmetric line) or four half-curves if $x_{0}^{*}<x_{0}<b$ ([a, $\left.\xi\right]$, $\left[\xi, x_{0}\right]$, the nodal line starting from $\xi$ and its symmetric).

Let $z_{0}^{+}$be the intersection point between the boundary $\partial \Omega \cap\{y>0\}$ and the nodal line joining $x_{0}^{*}$ to $\partial \Omega \cap\{y>0\}$. If the boundary $\partial \Omega \cap\{y>0\}$ is smooth at $z_{0}^{+}$, then the nodal line is orthogonal to the boundary at $z_{0}^{+}$. If $z_{0}^{+}$is a vertex of $\Omega$, then, locally around $z_{0}^{+}$, the nodal line splits the domain $\Omega$ in two domains with equal angles at $z_{0}^{+}$.

We now present results for several simple shapes: the square, the disk, and the union of three touching hexagons. The computations (see [4]) have been made with the Finite Element Library Mélina [20] using 6-order triangular elements, leading to accurate values (with relative error smaller than 0.01\%). More computations are available on the web page http://w3. bretagne.ens-cachan.fr/math/simulations/MinimalPartitions/form2.php.

Example 3.1 (the square). The two symmetries of the square (the axial symmetry through the middle of the two opposite sides or through the diagonal) provide two families of simulations.

Let us first use the axial symmetry mapping the upper-half square on the lower-half square. It turns out that for $x_{0}<(a+b) / 2$, the second eigenmode only generates a 2-partition of $\Omega$. Moreover for $x_{0}=(a+b) / 2$, the nodal line joins $x_{0}$ to the top boundary. Hence $x_{0}^{*}=(a+b) / 2$. Let us mention that for $x_{0}>x_{0}^{*}$, the nodal line starts from $\xi \in\left(a, x_{0}\right)$. Figure 10 illustrates these three cases. This figure also illustrates the equal angle meeting property. We first observe that the nodal line joining $x_{0}$ to the boundary $\partial \Omega \cap\{y>0\}$ is orthogonal to $\partial \Omega \cap\{y>0\}$. Secondly, the nodal line is orthogonal to $\left(a, x_{0}\right)$ if $x_{0}$ is different from $x_{0}^{*}$ and the angle between these two curves equals $2 \pi / 3$ when $x_{0}=x_{0}^{*}$. A candidate for the minimal 3 -partition of the square is given by the mixed problem for $x_{0}=(a+b) / 2$ according to the numerics.

We can proceed to similar computations using the diagonal symmetry, see Figure 11. The previous numerical strategy applied with this symmetry provides a new candidate for the minimal 3-partition of the square given by the mixed problem for $x_{0}=(a+b) / 2$. 


$$
x_{0}<x_{0}^{*}
$$
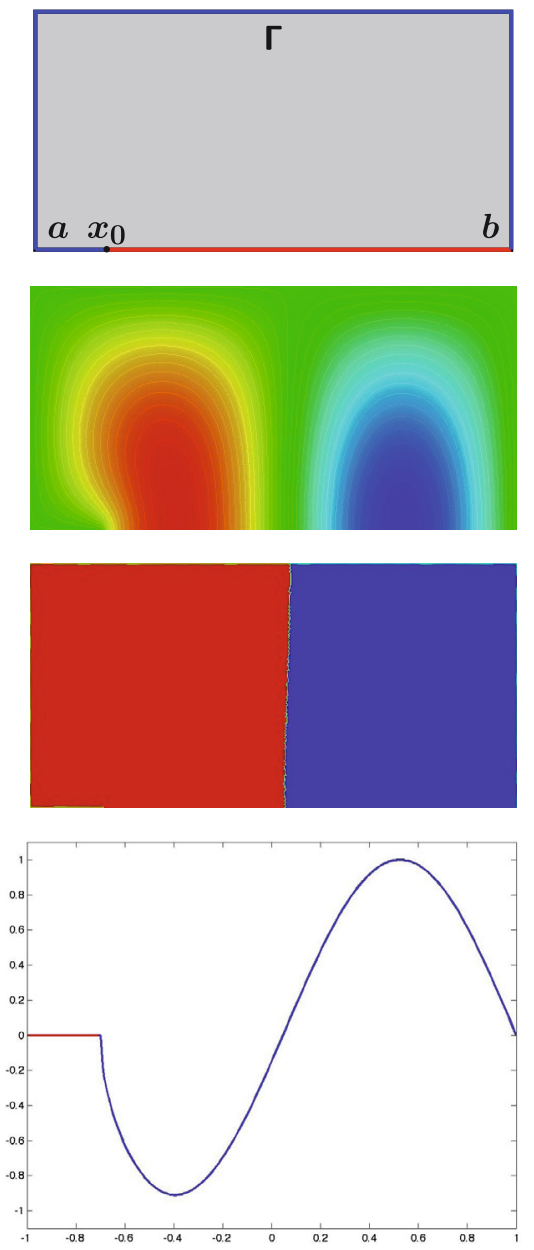

$$
x_{0}=x_{0}^{*}
$$
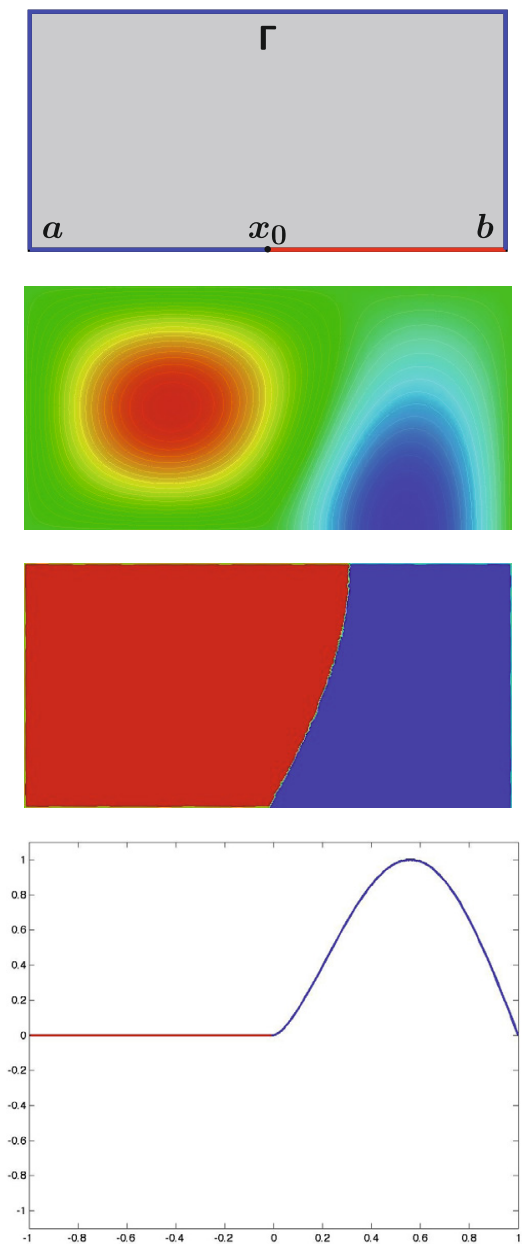

$x_{0}>x_{0}^{*}$
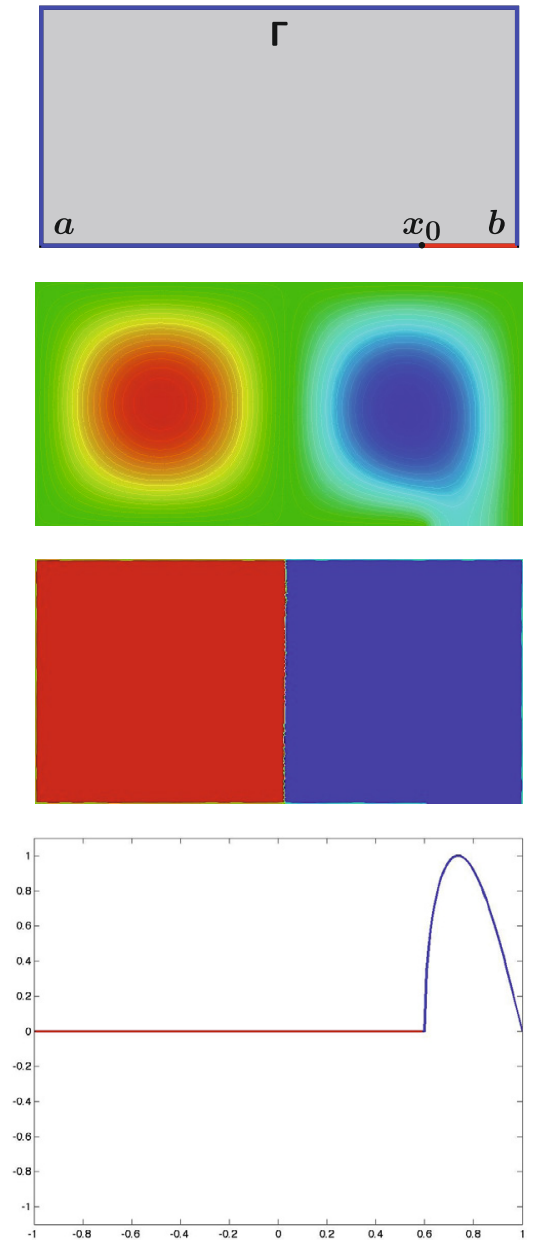

FIGURE 10. Eigenfunctions for the Dirichlet-Neumann problem on the half-square. First row: the domain; second row: the second eigenfunction $\varphi_{x_{0}}$; third row: the nodal domains of $\varphi_{x_{0}}$; fourth row: trace of $\varphi_{x_{0}}$ on $y=0$.

Table 1 shows that the second eigenvalues of the mixed problems with Dirichlet condition on the half horizontal line in the rectangle and the isoceles triangle constituting a half square are equal. Thus the numerical simulations provide two 3-partitions $\mathcal{D}_{0}$ and $\mathcal{D}_{1}$ of the square after symmetrization of the nodal sets of the second eigenfunction of the previous mixed problem with $x_{0}$ the middle point of the symmetry axis. These partitions are represented in the first two pictures of Figure 17 and satisfy

$$
\Lambda\left(\mathcal{D}_{0}\right)=\Lambda\left(\mathcal{D}_{1}\right)
$$

If we admit, as it is suggested by the numerical simulations but not proved, that the critical point of the minimal 3-partition is the middle point of the square, isospectral arguments proposed by Hoffmann-Ostenhof in a personal communication suggest that we have a continuum of minimal 3-partitions: there exists a continuous family $\left(\mathcal{D}_{t}\right)_{t \in[0,1]}$ with $\Lambda\left(\mathcal{D}_{t}\right)=\Lambda\left(\mathcal{D}_{0}\right)=\Lambda\left(\mathcal{D}_{1}\right)$ for any $t \in[0,1]$. For more details, we refer to $[13,17,19]$. 

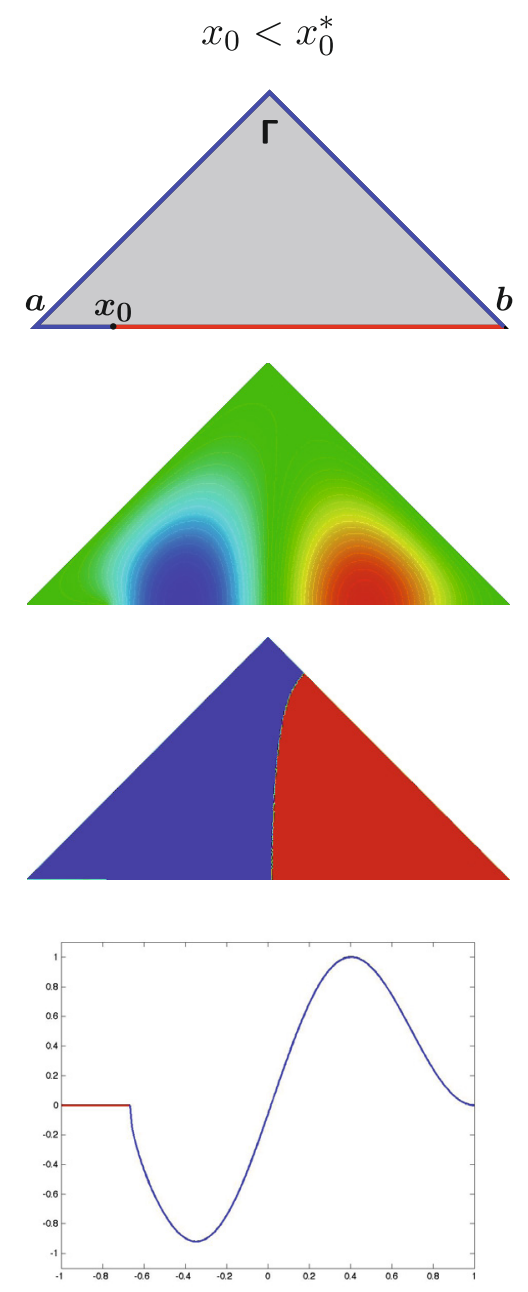

$x_{0}=x_{0}^{*}$
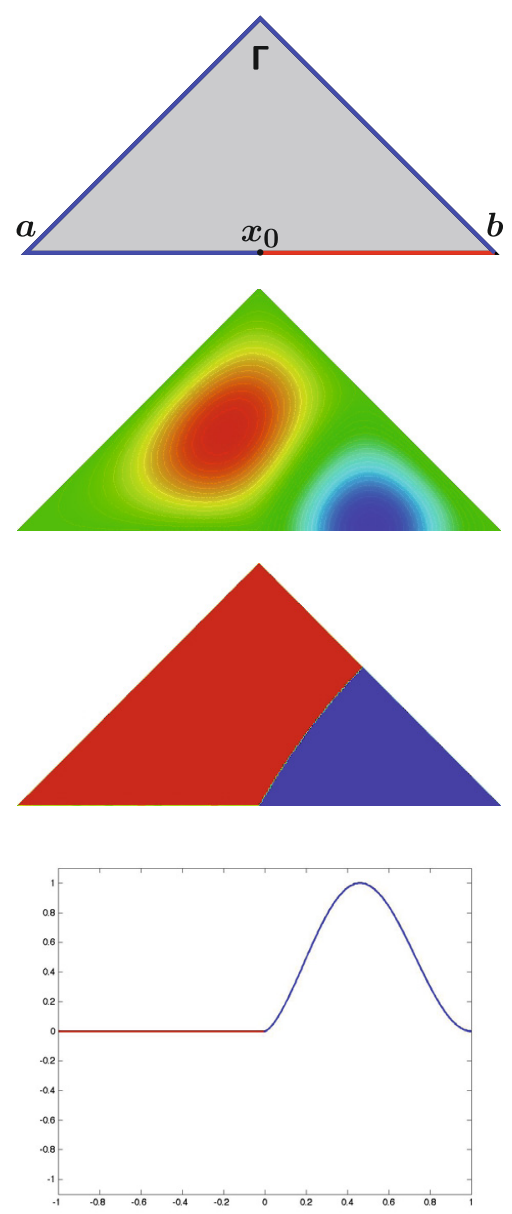

$x_{0}>x_{0}^{*}$
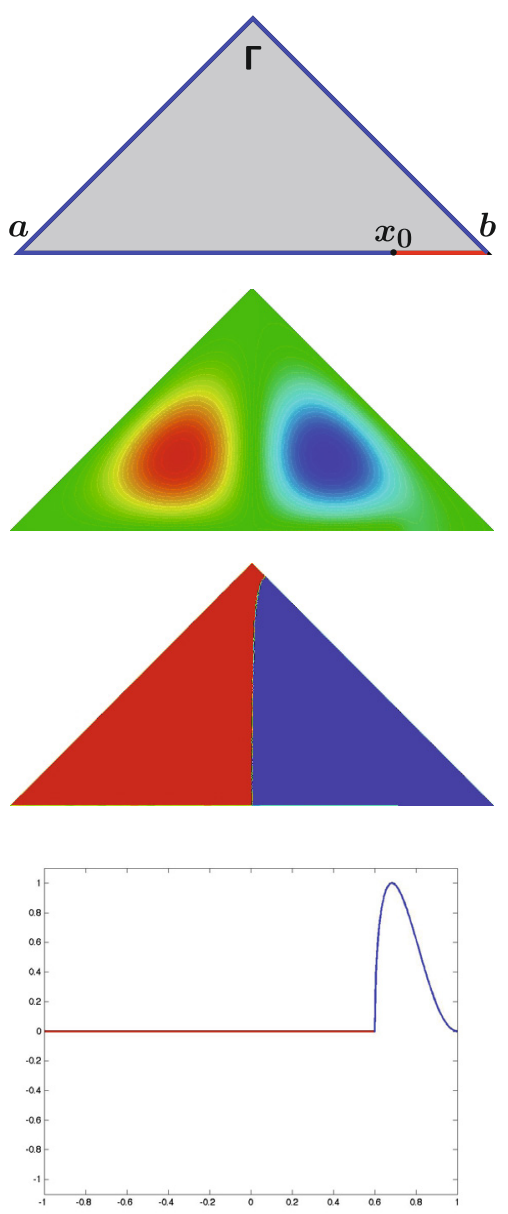

FIGURE 11. Eigenfunctions for the Dirichlet-Neumann problem on the half-square. First row: the domain; second row: the second eigenfunction $\varphi_{x_{0}}$; third row: the nodal domains of $\varphi_{x_{0}}$; fourth row: trace of $\varphi_{x_{0}}$ on $y=0$.

Example 3.2 (the disk). We recover the natural candidate (straight segment) for the disk, with $x_{0}^{*}$ at the center of the disk. The possible minimal 3-partition seems to be the family consisting of three identical sectors (see Fig. 12). The properties about the angle between the nodal line and the boundary $\partial \Omega \cap\{y>0\}$ or the line $\left(a, x_{0}\right)$ are the same as in the case of the square.

Example 3.3 (the union of three touching hexagons). The configuration built from $\varphi_{x_{0}^{*}}$ after symmetrization corresponds to the three hexagons composing the domain (see Fig. 13). Looking at Figure 13, we notice that the nodal line is orthogonal to the boundary $\partial \Omega \cap\{y>0\}$ as soon as $x_{0} \neq x_{0}^{*}$ and the angle between these two curves equals $2 \pi / 3$ when $x_{0}=x_{0}^{*}$.

Remark 3.1. In the previous examples, we have only computed the second eigenmodes of the mixed problems. One cannot exclude a priori that for some $x_{0}$, the third (or higher) eigenmode will provide a better configuration than $\left(\lambda_{2}\left(x_{0}^{*}\right), \varphi_{x_{0}^{*}}\right)$. However, we know that $\lambda_{3}\left(x_{0}\right) \geq \lambda_{3}(a)$ for any $x_{0} \in[a, b]$, and in the three examples tested 

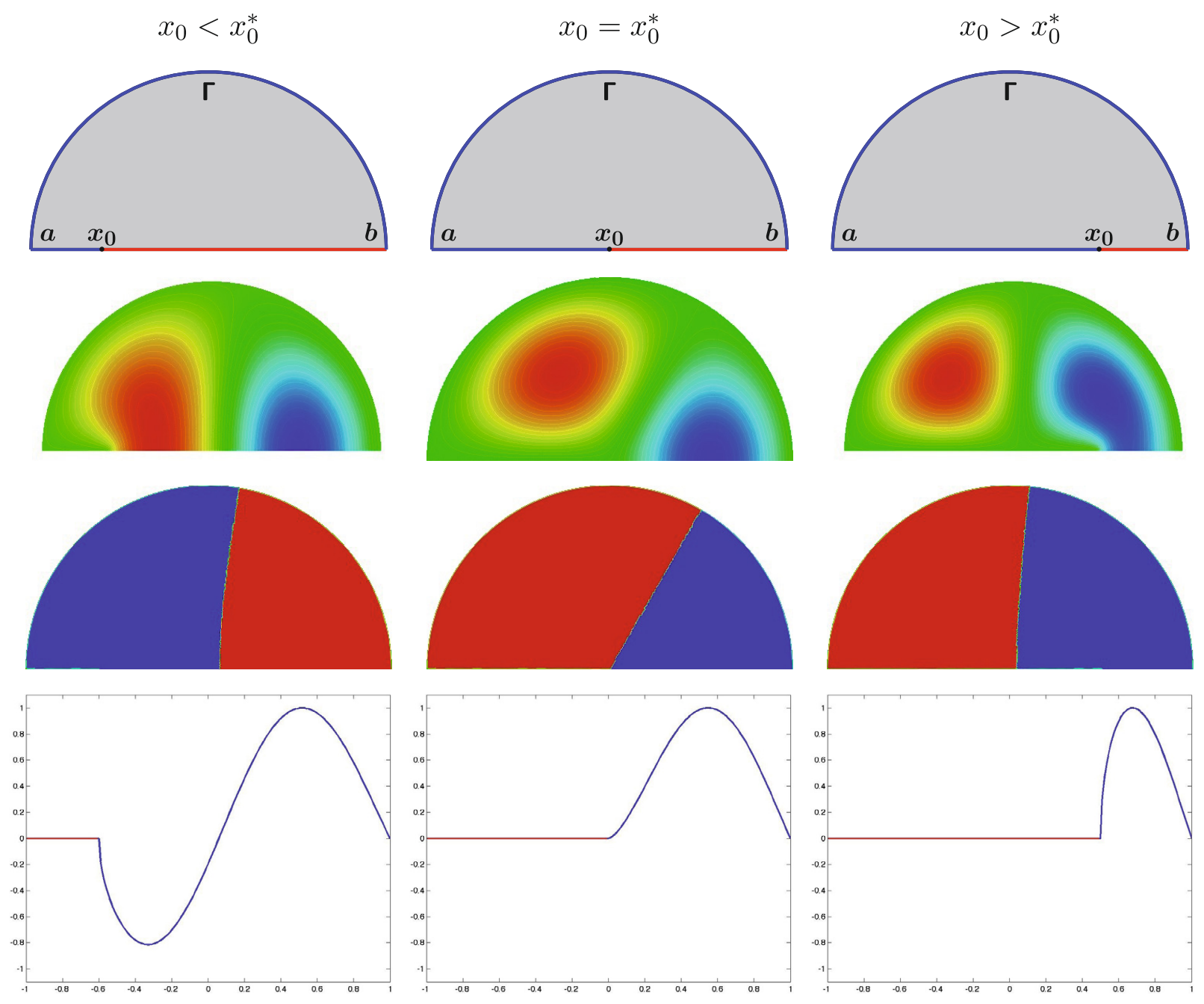

FIGURE 12. Eigenfunctions for the Dirichlet-Neumann problem on the half-disk. First row: the domain; second row: the second eigenfunction $\varphi_{x_{0}}$; third row: the nodal domains of $\varphi_{x_{0}}$; fourth row: trace of $\varphi_{x_{0}}$ on $y=0$.

TABLE 1. Numerical eigenvalues for the square, the disk and the 3-hexagons.

\begin{tabular}{|l|c|c|c|}
\hline & $x_{0}^{*}$ & $\lambda_{2}\left(x_{0}^{*}\right)$ & $\lambda_{3}(a)$ \\
\hline Square & $(a+b) / 2$ & 16.6453 & 24.6740 \\
\hline Square (diagonal) & $(a+b) / 2$ & 16.6453 & 19.7392 \\
\hline Disk & $(a+b) / 2$ & 20.1994 & 26.3860 \\
\hline 3-hexagons & $(a+b) / 3$ & 18.5901 & 27.5868 \\
\hline
\end{tabular}




$$
x_{0}<x_{0}^{*}
$$
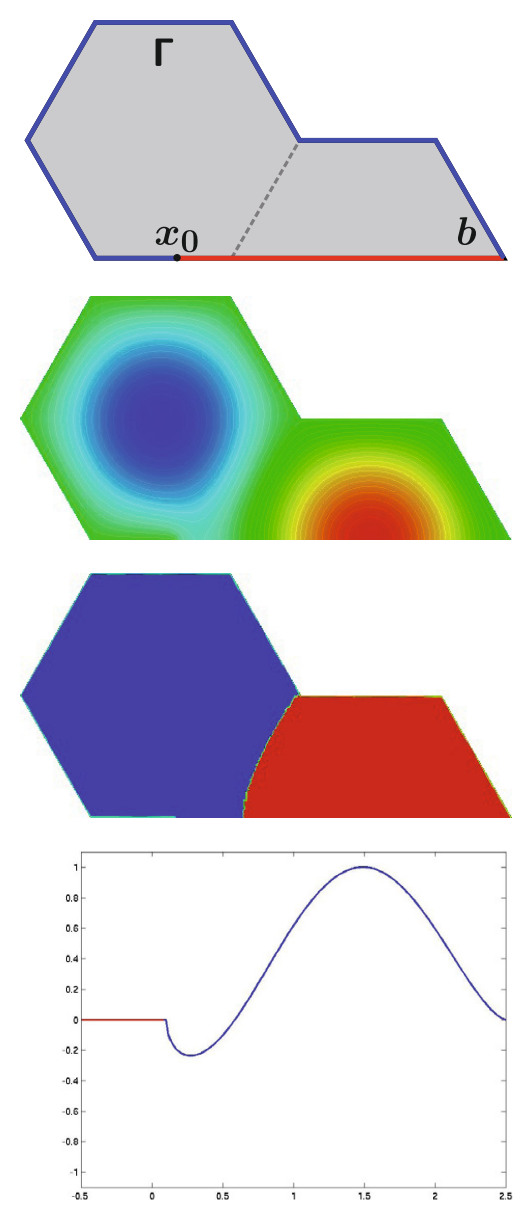

$$
x_{0}=x_{0}^{*}
$$
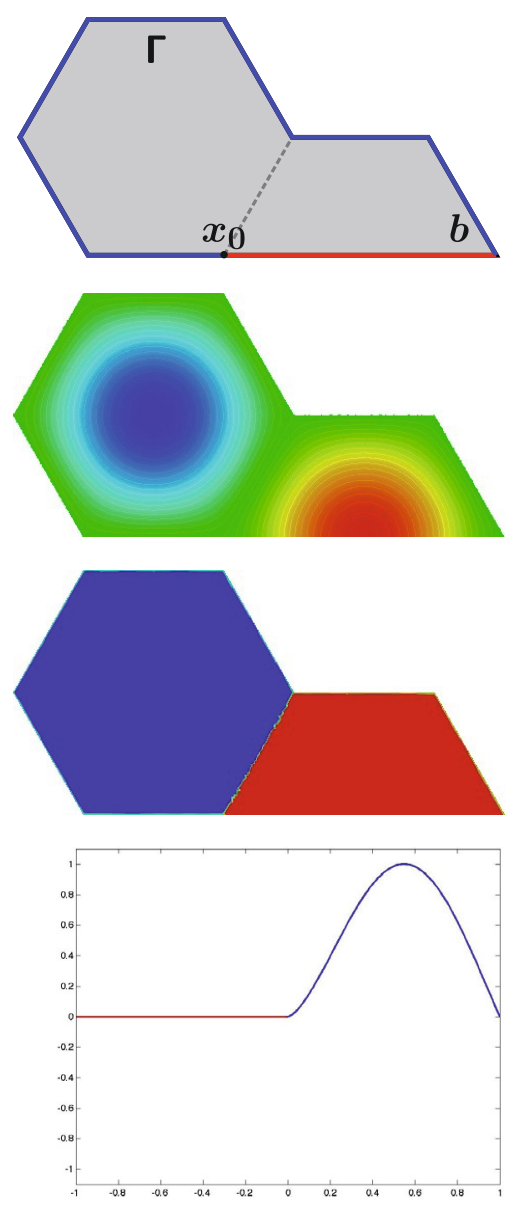

$x_{0}>x_{0}^{*}$
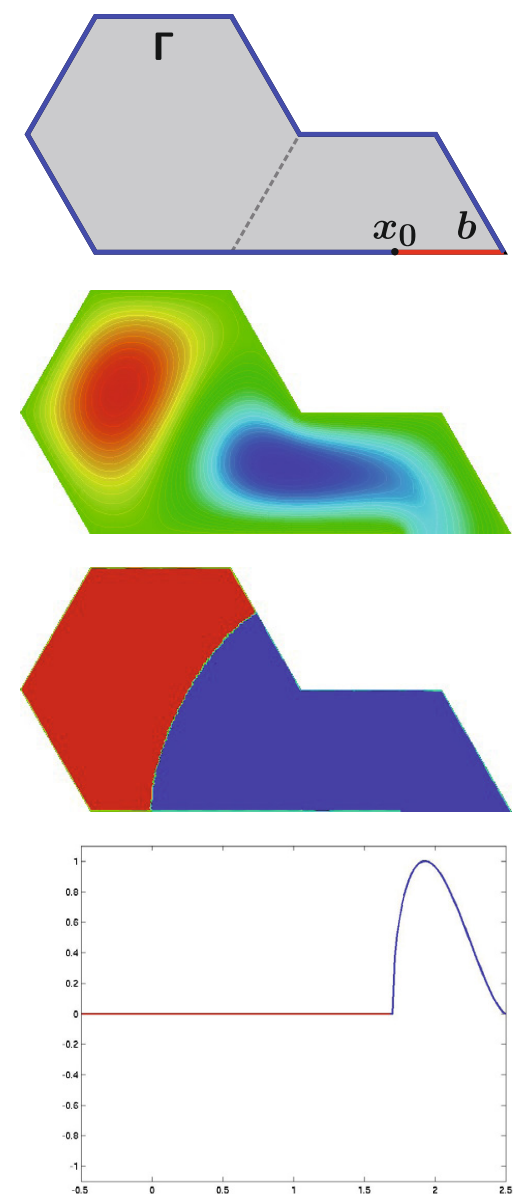

Figure 13. Eigenfunctions for the Dirichlet-Neumann problem on the half-three hexagons. First row: the domain; second row: the second eigenfunction $\varphi_{x_{0}}$; third row: the nodal domains of $\varphi_{x_{0}}$; fourth row: trace of $\varphi_{x_{0}}$ on $y=0$.

previously, computations show that $\lambda_{3}(a)>\lambda_{2}\left(x_{0}^{*}\right)$ (see Tab. 1). Consequently, only the second eigenmode can generate an interesting candidate.

\subsection{Non bipartite 3-partition with two critical points}

We focus here on cases (b) and (c), see Figures 7 and 8. The analysis of these two cases can be done similarly. This time we get a Dirichlet-Neumann-Dirichlet or Neumann-Dirichlet-Neumann condition on $[a, b]$ (see Fig. 14). We denote respectively by $\lambda_{k}^{D N D}\left(x_{0}, x_{1}\right)$ and $\lambda_{k}^{N D N}\left(x_{0}, x_{1}\right)$ the $k$-th eigenvalues of the mixed problem with Neumann conditions respectively on $\left[x_{0}, x_{1}\right]$ and $\left[a, x_{0}\right] \cup\left[x_{1}, b\right]$ and Dirichlet conditions elsewhere (cf. Fig. 14). 

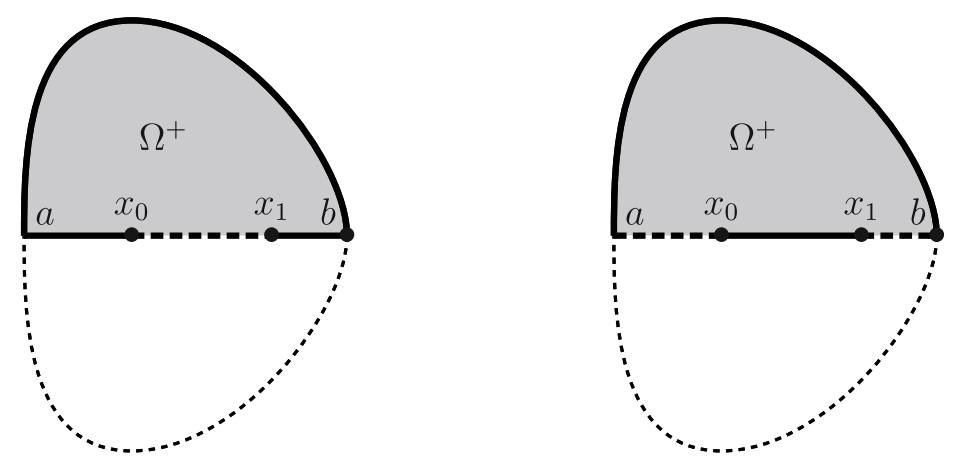

FiguRE 14. Dirichlet-Neumann-Dirichlet or Neumann-Dirichlet-Neumann problem.
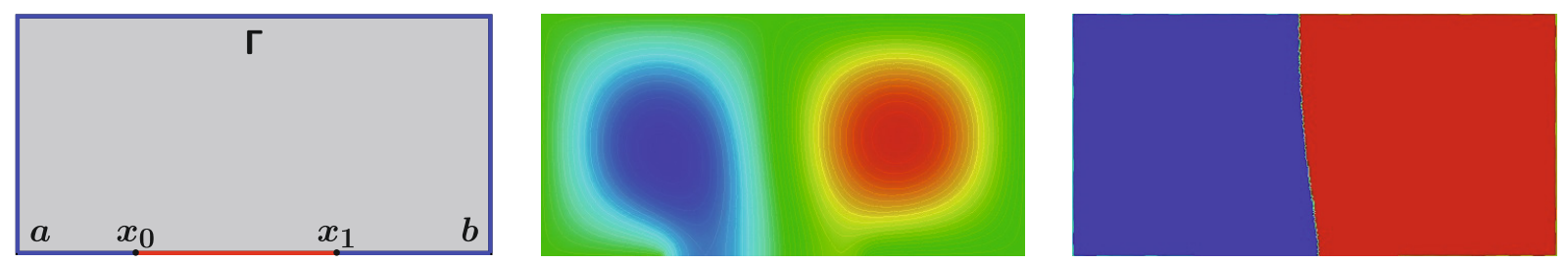

FiguRE 15. Eigenfunctions for the Dirichlet-Neumann-Dirichlet problem on the half-square.

Left: the domain; center: the second eigenfunction; right: the associated nodal domains.
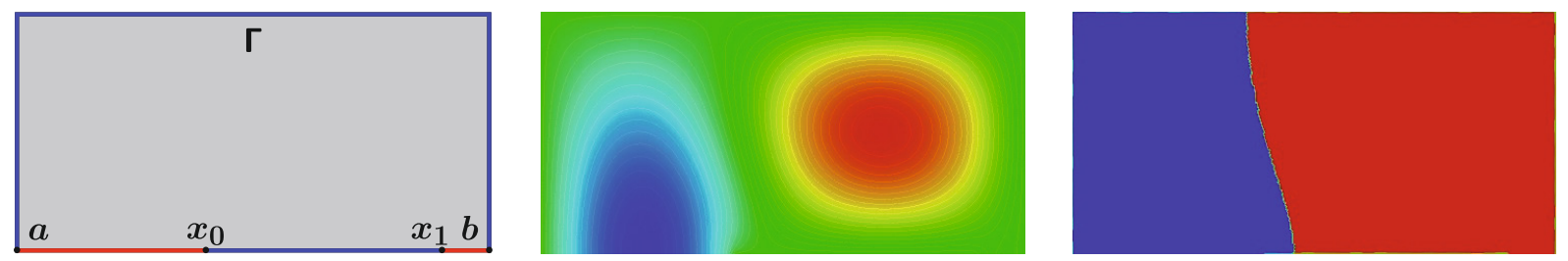

Figure 16. Eigenmodes for the Neumann-Dirichlet-Neumann problem on the half-square. Left: the domain; center: the second eigenfunction; right: the associated nodal domains.

Obviously, we have

$$
\lambda_{k}^{D N D}\left(x_{0}, x_{1}\right) \geq \lambda_{k}\left(x_{0}\right) \geq \lambda_{k}(a) \quad \text { and } \quad \lambda_{k}^{N D N}\left(x_{0}, x_{1}\right) \geq \lambda_{k}(a) .
$$

As in the case of the Dirichlet-Neumann condition, we compute the second eigenmode but numerical computations for the semi-square, the semi-disk and the semi-3-hexagon suggest that the nodal line of the second eigenfunction never creates a 2-partition of $\Omega^{+}$leading by symmetry to a 3 -partition of $\Omega$. Figures 15 and 16 give the eigenfunction associated with $\lambda_{2}^{D N D}\left(x_{0}, x_{1}\right)$ and $\lambda_{2}^{N D N}\left(x_{0}, x_{1}\right)$ respectively. Changing the parameters $x_{0}$ and $x_{1}$ does not change the configuration. We can look at the following modes to generate a better candidate for a 3-partition. As mentioned in (3.3), the third eigenvalue is always bounded from below by $\lambda_{3}(a)$. In the case of the square, the disk and the 3-hexagon, numerical estimates given in Table 1 show that $\lambda_{3}(a)$ is larger than $\lambda_{2}\left(x_{0}^{*}\right)$, the best "energy" obtained by the mixed problem Dirichlet-Neumann. Then, the only symmetric candidate is given by the Dirichlet-Neumann condition. 

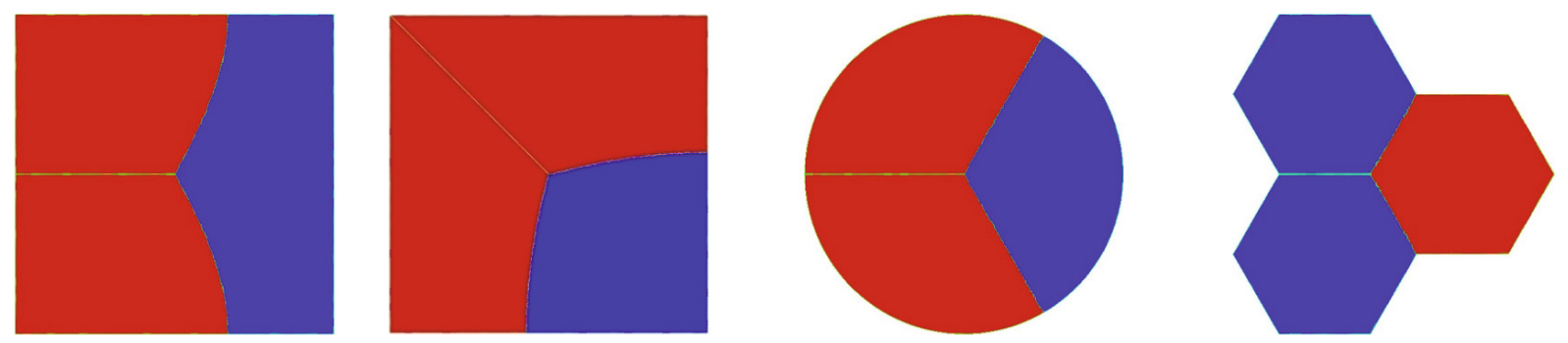

Figure 17. Candidates for the minimal 3-partitions.

\subsection{Conclusion}

Our numerical discussion provides candidates for each geometry given by case a (cases (b) and (c) do not exhibit any interesting configuration). After symmetrization, the associated 3-partitions are represented in Figure 17. Let us mention that the minimal 3-partition is not bipartite for the three considered shapes, otherwise the third eigenvector would be Courant-sharp (it is obviously not the case for the square and the disk, and nor for the three touching hexagons thanks to a numerical computation). The numerical simulations suggest that, if the minimal 3-partition is symmetric, it contains one critical point at the center for the square or the disk and at the intersection of the three touching hexagons for this geometry.

Other techniques are developed in [11] based on entropy considerations to provide candidates to minimize the $\operatorname{sum} \sum_{i=1}^{k} \lambda_{1}\left(D_{i}\right)$ with $k \geq 5$.

\section{Minimal partition AND TILINGS}

In this section, we are interested in the asymptotic behavior of $\mathfrak{L}_{k}(\Omega) / k$ as $k \rightarrow \infty$. In Section 4.1 , after recalling the Faber-Krahn inequality and properties of tilings ${ }^{6}$, we bound $\lim \sup \mathfrak{L}_{k}(\Omega) / k$ and $\lim \inf \mathfrak{L}_{k}(\Omega) / k$ by the first eigenvalue of the Dirichlet Laplacian on the disk and the hexagon of area 1 . We conjecture (see Conjectures 4.1 and 4.2$)$ that $|\Omega| \mathfrak{L}_{k}(\Omega) / k$ converges to the first eigenvalue of the hexagon as $k \rightarrow \infty$. The following part is devoted to the relevance of this conjecture. We prove it is true if there exists a minimal $\mathbb{R}^{2}$-partition (see Def. 4.5) which is a tiling. Then we discuss self-similar tilings with equilateral triangles or squares and at last propose simulations on tilings with hexagons.

\subsection{On the asymptotic behavior of $\mathfrak{L}_{k}(\Omega) / k$}

An interesting question was communicated to the authors of [16] by M. Van den Berg. We would like also to thank A. El Soufi for discussions around this problem. By the Faber-Krahn inequality it is easy to see that

$$
\lambda_{1}\left(\operatorname{Disk}_{1}\right) \leq|\Omega| \frac{\mathfrak{L}_{k}(\Omega)}{k}
$$

where $D_{i s k_{1}}$ is the disk of area 1 and $\Omega$ is a regular domain.

On the other hand, if one considers any tiling associated with a discrete group of isometries ${ }^{7}$ of $\mathbb{R}^{2}$ and if $D_{1}$ is the fundamental cell (which could be a square, a triangle or an hexagon), then we have asymptotically

\footnotetext{
${ }^{6}$ It is worth to mention that tilings appear in a connected subject in Pólya's work [23].

${ }^{7}$ We say that a strong partition $\mathcal{D}=\left(D_{i}\right)_{i}>1$ of $\mathbb{R}^{2}$ is a tiling (in French 'pavage') of $\mathbb{R}^{2}$ associated with $\Gamma$ if $\Gamma$ is a discrete group of isometries such that for any $D_{i} \in \mathcal{D}$, there exists $\gamma \in \Gamma$ with $D_{i}=\gamma D_{1}$, and such that $\gamma D_{1}=\gamma^{\prime} D_{1}$ implies $\gamma=\gamma^{\prime}$.
} 


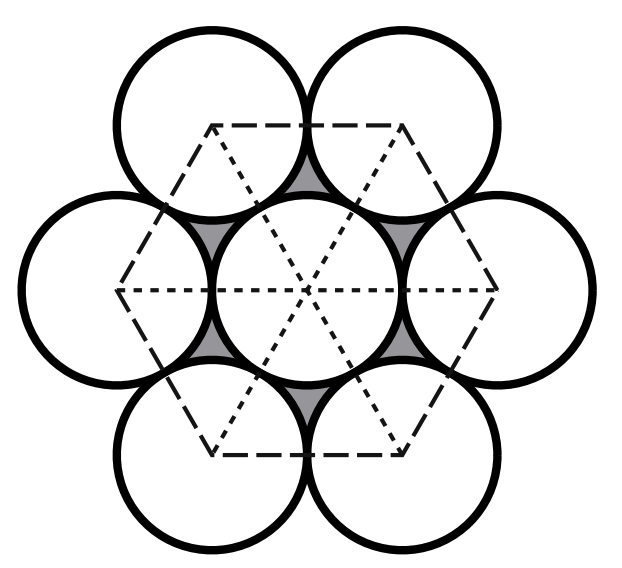

Figure 18. Tiling with disks.

the upper bound

$$
|\Omega| \limsup _{k \rightarrow+\infty} \frac{\mathfrak{L}_{k}(\Omega)}{k} \leq\left|D_{1}\right| \lambda_{1}\left(D_{1}\right) .
$$

Here are a few numerical (sometimes exact) values corresponding to the $H e x a_{1}, T_{1}$ and $S q_{1}$ being respectively a regular hexagon, an equilateral triangle and a square of area 1 . Then

$$
\lambda_{1}\left(\text { Hexa }_{1}\right) \sim 18.5901, \lambda_{1}\left(S q_{1}\right)=2 \pi^{2} \sim 19.7392, \lambda_{1}\left(T_{1}\right) \sim 22.7929
$$

Then, as it is well known, we observe that the lowest eigenvalue of the Dirichlet realization of the Laplacian on the regular hexagon of area 1 is lower than the ground state energy of the triangle or the square of same area.

Beside the ground state energy of the disk is

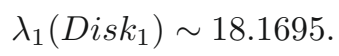

So we get

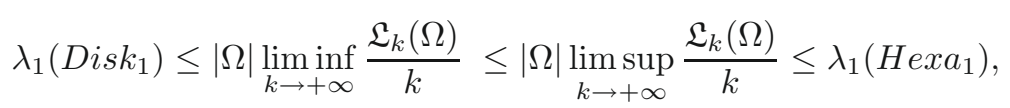

with

$$
\lambda_{1}\left(\text { Disk }_{1}\right)<\lambda_{1}\left(\text { Hexa }_{1}\right) .
$$

This leads to two conjectures.

Conjecture 4.1. The limit of $\mathfrak{L}_{k}(\Omega) / k$ as $k \rightarrow+\infty$ exists.

Actually this limit might be more explicit:

\section{Conjecture 4.2 .}

$$
|\Omega| \lim _{k \rightarrow+\infty} \frac{\mathfrak{L}_{k}(\Omega)}{k}=\lambda_{1}\left(\operatorname{Hexa}_{1}\right) .
$$

This last conjecture says in particular that the limit is independent of $\Omega$ if $\Omega$ is a regular domain.

Of course the optimality of the regular hexagonal tiling appears in various contexts in Physics. But we have at the moment no idea of any approach for proving this in our context. We will only explore numerically why this conjecture looks reasonable. 
Remark 4.3. The following argument shows that tiling with hexagons is better than with disks. Looking at Figure 18, tiling with disks generates holes in the domain. Let us give the area of these holes. Let $R$ be the radius of the considered disk. Then the area of a hole $\mathcal{A}(R)$ is equal to the difference between the area of an equilateral triangle $\mathcal{A}_{T}(R)$ of side $2 R$ and the half area of a disk $\mathcal{A}_{D}(R)$ of radius $R$. We compute easily that

$$
\mathcal{A}_{T}(R)=R^{2} \sqrt{3} \quad \text { and } \quad \mathcal{A}_{D}(R)=\pi R^{2} .
$$

Then

$$
\mathcal{A}(R)=\mathcal{A}_{T}(R)-\frac{1}{2} \mathcal{A}_{D}(R)=R^{2}\left(\sqrt{3}-\frac{\pi}{2}\right) .
$$

If we consider a disk of area one, then $R=1 / \sqrt{\pi}$ and then

$$
\mathcal{A}\left(\frac{1}{\sqrt{\pi}}\right)=\frac{\sqrt{3}}{\pi}-\frac{1}{2} \simeq 5.1329 \times 10^{-2} .
$$

The area of the hexagon drawn in Figure 18 equals $6 \sqrt{3} / \pi$ and the area of the pieces of disks inside this hexagon equals 3. Then, we have to compare

$$
\frac{\lambda_{1}\left(D i s k_{1}\right)}{3} \times \frac{6 \sqrt{3}}{\pi}=\frac{2 \sqrt{3}}{\pi} \lambda_{1}\left(\text { Disk }_{1}\right) \simeq 20.0347 \quad \text { and } \quad \lambda_{1}\left(\text { Hexa }_{1}\right) \simeq 18.5901 .
$$

It follows that the tiling with regular hexagons gives a lower energy than those with disks.

\subsection{Towards a definition of uniform minimal partition of $\mathbb{R}^{2}$}

Let us start from an infinite strong regular partition $\mathcal{D}$ of $\mathbb{R}^{2}$. Of course we are principally interested in tilings attached to a discrete group of isometries $\Gamma$ but it seems interesting to have a slightly more general notion. One could be interested for example in starting from a tiling and in considering a refined partition obtained by repartitioning each $D_{i}$ of the tiling and using a minimal $m$-partition of $D_{i}$.

Another possibility could be to create a fundamental 'molecule' $\mathcal{M}_{0}$ by gluing together a union of $m$ previous $D_{i}$, and then considering a new $m$-partition of this molecule.

To cover all these cases, we are led to introduce a notion of uniformity.

Definition 4.4. Let $\mathcal{D}=\left(D_{i}\right)_{i \in I}$, an open partition covering $\mathbb{R}^{2}$ (i.e. $D_{i} \cap D_{j}=\emptyset$ if $i \neq j$ and $\cup_{i \in I} \overline{D_{i}}=\mathbb{R}^{2}$ ). The partition $\mathcal{D}$ is called uniform if

- each $D_{i}$ is bounded;

- the partition is locally finite: any disk of $\mathbb{R}^{2}$ is contained in a finite union of $\overline{D_{i}}$ 's.

We can now state a natural definition of uniform minimal $\mathbb{R}^{2}$-partition:

Definition 4.5. An infinite strong regular uniform partition $\mathcal{D}$ of $\mathbb{R}^{2}$ is called a minimal $\mathbb{R}^{2}$-partition if, for any $k$, any connected subpartition $\left(D_{i}\right)_{i \in I_{k}}$ of cardinal $k=\left|I_{k}\right|$ is a minimal $k$-partition of $\mathcal{D}_{I_{k}}=\operatorname{Int}\left(\cup_{i \in I_{k}} \overline{D_{i}}\right)$.

We do not know if such partitions exist but they seem to be rather good candidates for an accurate upper bound in the problem above.

Remark 4.6. Note that any open set $D_{i}$ of a minimal $\mathbb{R}^{2}$-partition has by definition the same groundstate $\lambda_{1}\left(D_{i}\right)$.

We now want to estimate $\mathfrak{L}_{k}(\Omega)$. Precisely, we prove the following result: 
Proposition 4.7. Suppose that there exists a minimal $\mathbb{R}^{2}$-partition $\mathcal{D}$ which is a tiling, then $\mathfrak{L}_{k}(\Omega) / k$ tends to a limit given by:

$$
|\Omega| \lim _{k \rightarrow+\infty} \frac{\mathfrak{L}_{k}(\Omega)}{k}=\left|D_{1}\right| \lambda_{1}\left(D_{1}\right)
$$

Remark 4.8. In particular if we show that the hexagonal tiling is a minimal $\mathbb{R}^{2}$-partition, we get that $\lambda_{1}\left(H e x a_{1}\right)$ is effectively the limit of $|\Omega| \mathfrak{L}_{k}(\Omega) / k$.

Our proof is based on the dilations $\delta_{R}: x \mapsto R x$ for any $R>0$ and requires some notation. For any non empty regular bounded open set of finite area $\Omega$, we introduce two subsets of $I: I^{\text {int }}(R, \Omega)$ and $I^{\text {ext }}(R, \Omega)$, defined by

$$
I^{\text {int }}(R, \Omega)=\left\{i \in I \mid D_{i} \subset \delta_{R} \Omega\right\}
$$

and

$$
I^{\text {ext }}(R, \Omega)=I^{\text {int }}(R, \Omega) \cup I^{\text {bnd }}(R, \Omega)
$$

where

$$
I^{\mathrm{bnd}}(R, \Omega)=\left\{i \in I \mid \partial \delta_{R} \Omega \cap D_{i} \neq \emptyset\right\}
$$

Remark 4.9. By uniformity (see Def. 4.4) it is clear that $\# I^{\operatorname{ext}}(R, \Omega)<+\infty$, for any bounded regular $\Omega$. Moreover in the case of a tiling, one has the following properties

$$
\# I^{\mathrm{int}}(R, \Omega) \sim R^{2} \frac{|\Omega|}{\left|D_{1}\right|}, \quad \text { and } \quad \# I^{\mathrm{bnd}}(R, \Omega)=\mathcal{O}(R) .
$$

The proof of Proposition 4.7 splits into two lemmas.

Lemma 4.10. Let $\mathcal{D}$ a uniform $\mathbb{R}^{2}$-partition (see Def. 4.4) satisfying

$$
\lambda_{1}\left(D_{i}\right)=\lambda_{1}\left(D_{j}\right), \forall i, j,
$$

then we have the upper bound

$$
\limsup _{k \rightarrow+\infty} \frac{\mathfrak{L}_{k}(\Omega)}{k} \leq \limsup _{k \rightarrow+\infty} \frac{R^{\mathrm{int}}(k, \Omega)^{2}}{k} \lambda_{1}\left(D_{i}\right)
$$

Proof. We start from

$$
\mathfrak{L}_{k}(\Omega)=R^{2} \mathfrak{L}_{k}\left(\delta_{R} \Omega\right)
$$

If $R$ satisfies

$$
\# I^{\text {int }}(R, \Omega) \geq k
$$

we obtain

$$
\mathfrak{L}_{k}(\Omega) \leq R^{2} \lambda_{1}\left(D_{i}\right)
$$

The optimal $R^{\text {int }}(k, \Omega)$ is given by

$$
R^{\text {int }}(k, \Omega):=\inf \left\{R \mid \# I^{\text {int }}(R, \Omega) \geq k\right\}
$$

Since $k \mapsto R^{\mathrm{int}}(k, \Omega)$ is monotonically increasing, we get the upper bound

$$
\frac{\mathfrak{L}_{k}(\Omega)}{k} \leq \frac{R^{\mathrm{int}}(k, \Omega)^{2}}{k} \lambda_{1}\left(D_{i}\right)
$$

Passing to the limit, we obtain the stated result. 
Remark 4.11. When $\mathcal{D}$ is a tiling, then we can obtain that

$$
|\Omega| \limsup _{k \rightarrow+\infty} \frac{R^{\text {int }}(k, \Omega)^{2}}{k}=\left|D_{1}\right| .
$$

In general, it is not the case, especially when the $D_{i}$ have not the same area. Nevertheless, the result can be extended when the partition can be clustered into molecules of same area: $I=\cup_{p \in J} I_{p}$ with $\left|I_{p}\right|=m$ and the molecules $\mathcal{M}_{p}=\operatorname{Int}\left(\cup_{i \in I_{p}} \overline{D_{i}}\right)$ of area $\left|\mathcal{A}_{0}\right|$. Indeed, if $\left(\mathcal{M}_{p}\right)_{p \in J}$ is a tiling associated with a discrete group, we obtain

$$
|\Omega| \limsup _{k \rightarrow+\infty} \frac{R^{\text {int }}(k, \Omega)^{2}}{k}=\frac{\left|\mathcal{A}_{0}\right|}{m} .
$$

Lemma 4.12. Under the assumptions of Lemma 4.10, we have the lower bound

$$
\liminf _{k \rightarrow+\infty} \frac{\mathfrak{L}_{k}(\Omega)}{k} \geq \liminf _{k \rightarrow+\infty} \frac{R^{\operatorname{ext}}(k, \Omega)^{2}}{k} \lambda_{1}\left(D_{1}\right) .
$$

Proof. We define

$$
R^{\text {ext }}(k, \Omega)=\sup \left\{R \mid \# I^{\text {ext }}(R, \Omega) \leq k\right\} .
$$

It is easy to see that $k \mapsto R^{\text {ext }}(k, \Omega)$ is increasing and satisfies

$$
R^{\text {ext }}(k, \Omega) \leq R^{\text {int }}(k, \Omega) .
$$

Let us look at the lower bound. We have, for $R \leq R^{\operatorname{ext}}(k, \Omega)$,

$$
\mathfrak{L}_{k}(\Omega)=R^{2} \mathfrak{L}_{k}\left(\delta_{R} \Omega\right) \geq R^{2} \mathfrak{L}_{\# I^{\operatorname{ext}}(R, \Omega)}\left(\mathcal{D}_{I^{\operatorname{ext}}(R, \Omega)}\right)
$$

Here we have simply used the domain monotonicity of $\mathfrak{L}_{k}$.

Since the partition is minimal (This is the first time that we use fully this property!), we observe that

$$
\mathfrak{L}_{\# I^{\operatorname{ext}}(R, \Omega)}\left(\mathcal{D}_{I^{\operatorname{ext}}(R, \Omega)}\right)=\lambda_{1}\left(D_{1}\right)
$$

We then obtain

$$
\mathfrak{L}_{k}(\Omega) \geq R^{\operatorname{ext}}(k, \Omega)^{2} \lambda_{1}\left(D_{1}\right)
$$

whence the result.

Proof of Proposition 4.7. Recalling Remark 4.11, we get

$$
|\Omega| \liminf _{k \rightarrow+\infty} \frac{R^{\operatorname{ext}}(k, \Omega)^{2}}{k}=\left|D_{1}\right|=|\Omega| \limsup _{k \rightarrow+\infty} \frac{R^{\text {int }}(k, \Omega)^{2}}{k} .
$$

The conclusion follows immediately from Lemmas 4.10 and 4.12.

Remark 4.13. More generally, if $\mathcal{D}$ is not a tiling, the condition (weak regularity)

$$
\liminf _{k \rightarrow+\infty} \frac{R^{\mathrm{ext}}(k, \Omega)^{2}}{k}=\limsup _{k \rightarrow+\infty} \frac{R^{\mathrm{int}}(k, \Omega)^{2}}{k}
$$

still gives existence of a limit for $\mathfrak{L}_{k}(\Omega) / k$. 


\subsection{About self-similar tilings}

We establish that the assumption of Proposition 4.7 is not fulfilled for bipartite self-similar tilings and illustrate consequences by numerical simulations on self-similar equilateral triangles and squares. By self-similar we mean that there exists some integer $m>1$ such that $\delta_{m} D_{1}$ is a union of $m^{2}$ open sets of the initial tiling. For example the square is covered by four squares, the equilateral triangle can also be written as the union of four triangles.

Theorem 4.14. A bipartite self-similar tiling is never minimal in the sense of Definition 4.5.

Proof. Let $D_{1}$ be a fundamental cell assumed of area 1 . We wonder if there exists an integer $n \geq 1$ such that $\mathfrak{L}_{m^{2 n}}\left(\delta_{m^{n}} D_{1}\right)<\lambda_{1}\left(D_{1}\right)$. If not, we have for any $n \geq 1$,

$$
\mathfrak{L}_{m^{2 n}}\left(\delta_{m^{n}} D_{1}\right)=\lambda_{1}\left(D_{1}\right)
$$

Using the eigenvector associated with $\lambda_{1}\left(D_{1}\right)$, we can construct an eigenvector with $m^{2 n}$ nodal sets for the bipartite domain $\delta_{m^{n}} D_{1}$. From (4.10), it follows that the partition is nodal and hence Courant-sharp by Theorem 2.6. Then

$$
\lambda_{1}\left(D_{1}\right)=\mathfrak{L}_{m^{2 n}}\left(\delta_{m^{n}} D_{1}\right)=\lambda_{m^{2 n}}\left(\delta_{m^{n}} D_{1}\right) .
$$

By a scaling argument, we have

$$
\lambda_{m^{2 n}}\left(\delta_{m^{n}} D_{1}\right)=\frac{\lambda_{m^{2 n}}\left(D_{1}\right)}{m^{2 n}} .
$$

We recall the Weyl's asymptotics (this is the same argument as for Pleijel's theorem, see [22])

$$
\lambda_{k}(\Omega) \sim 4 \pi \frac{k}{|\Omega|} \quad \text { as } \quad k \rightarrow+\infty
$$

Applying this asymptotics with $k=m^{2 n}$ and $\Omega=D_{1}$ and (4.11), we get

$$
\lambda_{m^{2 n}}\left(\delta_{m^{n}} D_{1}\right)=\frac{\lambda_{m^{2 n}}\left(D_{1}\right)}{m^{2 n}} \sim 4 \pi \frac{m^{2 n}}{\left|D_{1}\right|} \frac{1}{m^{2 n}}=4 \pi \quad \text { as } \quad n \rightarrow+\infty .
$$

This leads to a contradiction since $4 \pi<\lambda_{1}\left(D i s k_{1}\right)<\lambda_{1}\left(D_{1}\right)$.

Remark 4.15. Note that the regular hexagonal tiling is not self-similar.

Let us illustrate Theorem 4.14 with numerical simulations on equilateral triangles and squares. Let $T_{1}$ and $S q_{1}$ be respectively an equilateral triangle and a square of area 1 . From $4^{n}$ patterns $T_{1}$ or $S q_{1}$, we construct new equilateral triangles and squares of area $4^{n}$ denoted by $T_{4^{n}}$ and $S q_{4^{n}}$. To illustrate Theorem 4.14, we compute the first 24 eigenmodes for $T_{1}, T_{4}, T_{16}$ and $S q_{1}, S q_{4}, S q_{16}$ by using the Finite Element Library Mélina [20]. These computations are available on [4]. Table 2 gives the numerical eigenvalues for these domains. Figures 19 and 20 represent some eigenfunctions and their corresponding nodal domains.

We notice that that the fourth eigenfunctions on $T_{4}$ and $S q_{4}$ are Courant-sharp and then we have

$$
\mathfrak{L}_{4}\left(T_{4}\right)=\lambda_{4}\left(T_{4}\right)=\lambda_{1}\left(T_{1}\right) \quad \text { and } \quad \mathfrak{L}_{4}\left(S q_{4}\right)=\lambda_{4}\left(S q_{4}\right)=\lambda_{1}\left(S q_{1}\right) .
$$

If the self-similar tiling associated with $T_{1}$ or $S q_{1}$ were minimal, then we would have

$$
\lambda_{16}\left(T_{16}\right)=\lambda_{1}\left(T_{1}\right) \quad \text { and } \quad \lambda_{16}\left(S q_{16}\right)=\lambda_{1}\left(S q_{1}\right) .
$$

We observe that the 16 th eigenvalues on $T_{16}$ and $S q_{16}$ are strictly less than $\lambda_{1}\left(T_{1}\right)$ and $\lambda_{1}\left(S q_{1}\right)$. This is in agreement with Theorem 4.14. If we look at the following eigenfunctions, we see that $\lambda_{22}\left(T_{16}\right)=\lambda_{1}\left(T_{1}\right)$ and 
TABLE 2. Numerical eigenvalues of $T_{1}, T_{4}, T_{16}$ and $S q_{1}, S q_{4}, S q_{16}$.

\begin{tabular}{|l|c|c|c||c|c|c|}
\hline$k$ & $\lambda_{k}\left(T_{1}\right)$ & $\lambda_{k}\left(T_{4}\right)$ & $\lambda_{k}\left(T_{16}\right)$ & $\lambda_{k}\left(S q_{1}\right)$ & $\lambda_{k}\left(S q_{4}\right)$ & $\lambda_{k}\left(S q_{16}\right)$ \\
\hline $\mathbf{1}$ & $\mathbf{2 2 . 7 9 2 9}$ & 5.6982 & 1.4246 & $\mathbf{1 9 . 7 3 9 2}$ & 4.9348 & 1.2337 \\
2 & 53.1834 & 13.2958 & 3.3240 & 49.3480 & 12.3370 & 3.0843 \\
3 & 53.1834 & 13.2958 & 3.3240 & 49.3480 & 12.3370 & 3.0843 \\
$\mathbf{4}$ & 91.1715 & $\mathbf{2 2 . 7 9 2 9}$ & 5.6982 & 78.9568 & $\mathbf{1 9 . 7 3 9 2}$ & 4.9348 \\
5 & 98.7692 & 24.6923 & 6.1731 & 98.6960 & 24.6740 & 6.1685 \\
6 & 98.7692 & 24.6923 & 6.1731 & 98.6960 & 24.6740 & 6.1685 \\
7 & 144.3549 & 36.0887 & 9.0222 & 128.3049 & 32.0762 & 8.0191 \\
8 & 144.3549 & 36.0887 & 9.0222 & 128.3049 & 32.0762 & 8.0191 \\
9 & 159.5502 & 39.8875 & 9.9719 & 167.7833 & 41.9458 & 10.4865 \\
10 & 159.5502 & 39.8875 & 9.9719 & 167.7833 & 41.9458 & 10.4865 \\
11 & 205.1360 & 51.2840 & 12.8210 & 177.6530 & 44.4132 & 11.1033 \\
12 & 212.7336 & 53.1834 & 13.2959 & 197.3921 & 49.3480 & 12.3370 \\
13 & 212.7336 & 53.1834 & 13.2959 & 197.3921 & 49.3480 & 12.3370 \\
14 & 235.5265 & 58.8816 & 14.7204 & 246.7401 & 61.6850 & 15.4213 \\
15 & 235.5265 & 58.8816 & 14.7204 & 246.7401 & 61.6850 & 15.4213 \\
$\mathbf{1 6}$ & 281.1122 & 70.2781 & $\mathbf{1 7 . 5 6 9 5}$ & 256.6097 & 64.1524 & $\mathbf{1 6 . 0 3 8 1}$ \\
17 & 281.1123 & 70.2781 & 17.5695 & 256.6097 & 64.1524 & 16.0381 \\
18 & 296.3075 & 74.0769 & 18.5192 & 286.2185 & 71.5546 & 17.8887 \\
19 & 296.3075 & 74.0769 & 18.5192 & 286.2185 & 71.5546 & 17.8887 \\
20 & 326.6980 & 81.6745 & 20.4186 & 315.8273 & 78.9568 & $\mathbf{1 9 . 7 3 9 2}$ \\
21 & 326.6980 & 81.6745 & 20.4186 & 335.5666 & 83.8916 & 20.9729 \\
22 & 364.6862 & 91.1715 & $\mathbf{2 2 . 7 9 2 9}$ & 335.5666 & 83.8916 & 20.9729 \\
23 & 372.2838 & 93.0709 & 23.2677 & 365.1754 & 91.2938 & 22.8235 \\
24 & 372.2838 & 93.0709 & 23.2677 & 365.1754 & 91.2938 & 22.8235 \\
\hline
\end{tabular}

$$
\lambda_{1}\left(T_{1}\right)=22.7929
$$

$$
\lambda_{4}\left(T_{4}\right)=22.7929
$$

$$
\lambda_{16}\left(T_{16}\right)=17.5695
$$

$\lambda_{22}\left(T_{16}\right)=22.7929$
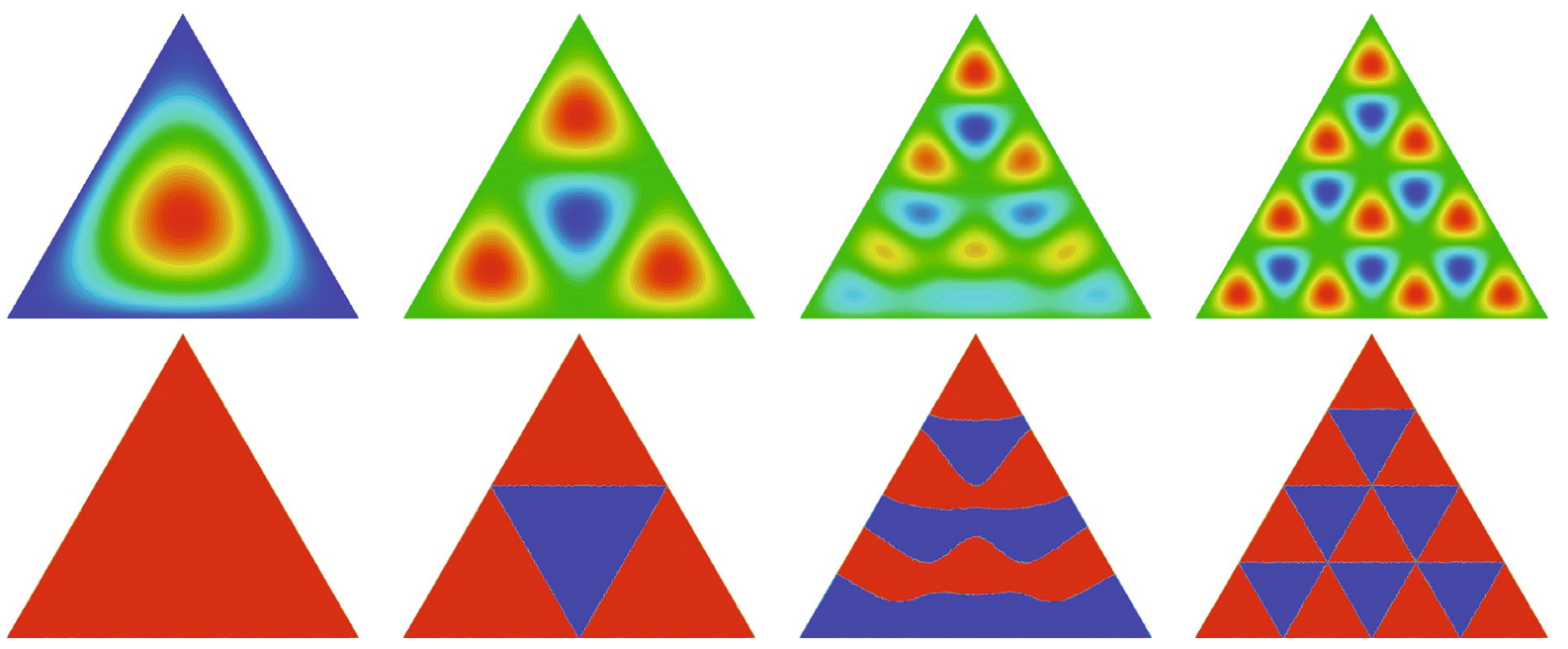

FIGURE 19. Eigenfunctions associated with $\lambda_{1}\left(T_{1}\right), \lambda_{4}\left(T_{4}\right), \lambda_{16}\left(T_{16}\right), \lambda_{22}\left(T_{16}\right)$. Top: eigenvalues; middle: eigenfunctions; bottom: the associated nodal domains. 

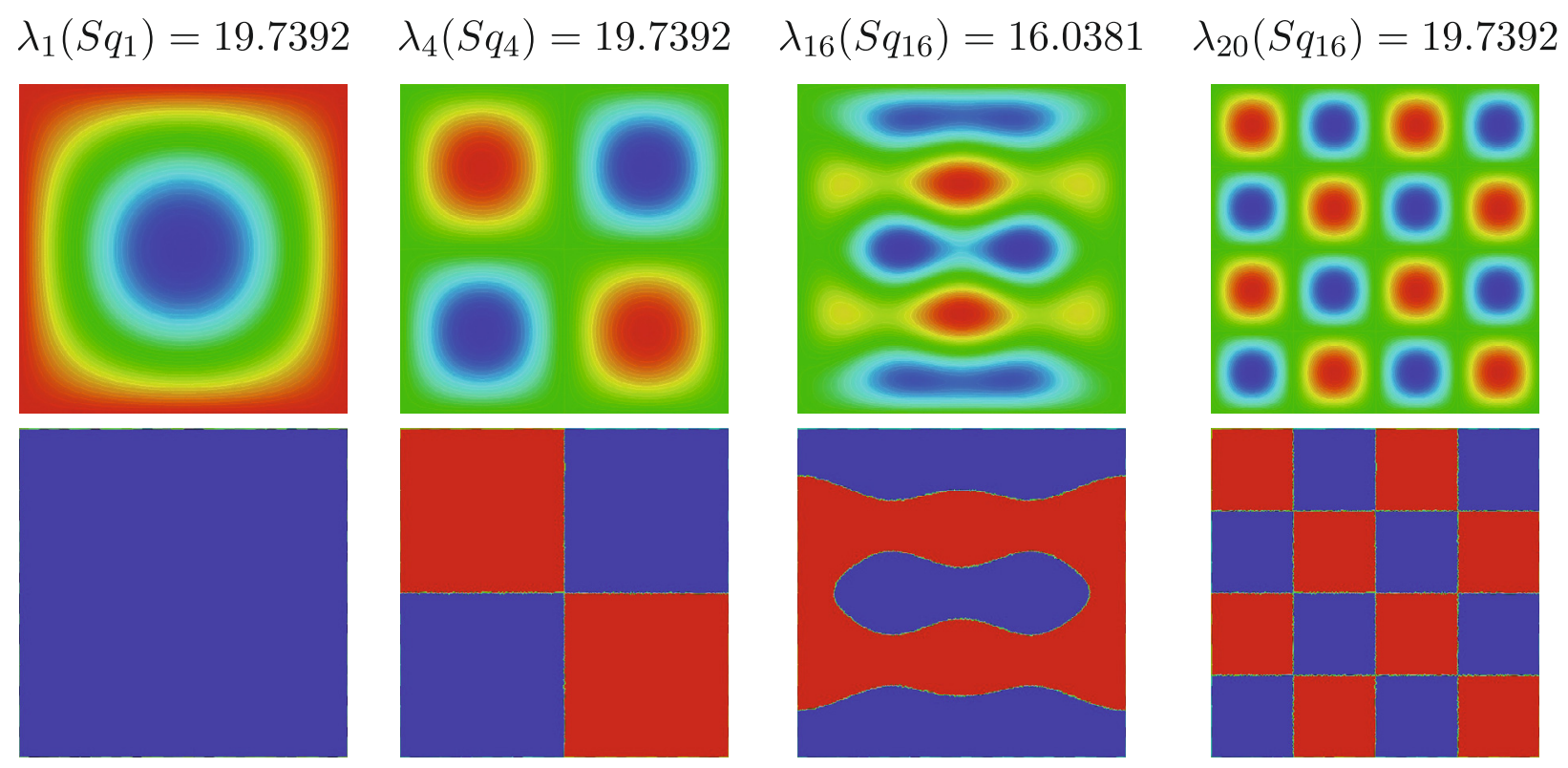

Figure 20. Eigenfunctions associated with $\lambda_{1}\left(S q_{1}\right), \lambda_{4}\left(S q_{4}\right), \lambda_{16}\left(S q_{16}\right), \lambda_{20}\left(S q_{16}\right)$. Top: eigenvalues; middle: eigenfunctions; bottom: the associated nodal domains.

the 22 th eigenfunction on $T_{16}$ has 16 nodal domains. For the square, the same situation appears for the 20 th eigenmode of $S q_{16}$.

Let us mention that for the square, the eigenmodes are explicit. The eigenvalues of a square of side $a$ are given by

$$
\mu_{j, k}=\frac{\pi^{2}}{a^{2}}\left(j^{2}+k^{2}\right), \quad j \geq 1, k \geq 1
$$

This formula can confirm the accuracy of the computations given in Table 2: the error is less than $10^{-4}$. The 16-th eigenvalue on $S q_{16}$ is double and equals $13 \pi^{2} / 8$. Any linear combination of $(x, y) \mapsto \sin (5 \pi x) \sin (\pi y)$ and $(x, y) \mapsto \sin (\pi x) \sin (5 \pi y)$ is an eigenfunction for $\lambda_{16}\left(S q_{16}\right)$. In the case of the triangle, we do not have any explicit formula. Nevertheless, the computations show some multiple eigenvalues: in Table 2, each pair of numerically-close eigenvalues effectively corresponds to a double eigenvalue since the nodal sets of the associated eigenfunctions do not satisfy the symmetry properties of the domain. Indeed, if one eigenvalue is simple, the new eigenfunction obtained after symmetry is colinear with the considered eigenfunction. For example, the values $\lambda_{16}$ and $\lambda_{17}$, given in Table 2, are numerically close and Figures 19 and 20 show that the eigenfunction associated with $\lambda_{16}$ does not satisfy the symmetry property, so the eigenvalue $\lambda_{16}$ is double.

In conclusion, the presented computations on the triangle and the square corroborate Theorem 4.14, showing that a bipartite self-similar tiling is not minimal. Furthermore, the numerics exhibit a value of $n$ such that $\mathfrak{L}_{m^{2 n}}\left(\delta_{m^{n}} D_{1}\right)<\lambda_{1}\left(D_{1}\right)$ for $m=2$ and 4 whereas the theoretical proof does not provide any explicit value since based on asymptotic tools.

\subsection{Hexagonal tilings}

\subsubsection{Playing with hexagons}

The previous result does not apply to the regular hexagonal tiling since it is not self-similar as already mentioned. In this section, we investigate its properties. In order to explore the Conjectures 4.1 and 4.2 , we have to check the weaker following one. 
Conjecture 4.16. For given $k \in \mathbb{N}$, we consider the family of open connected sets $\mathcal{H}^{(k)}$ which are unions of $k$ hexagons of area 1. Then

$$
\forall \Omega \in \mathcal{H}^{(k)}, \mathfrak{L}_{k}(\Omega)=\lambda_{1}\left(H e x a_{1}\right) .
$$

In other words, the $k$-partition of $\Omega$ by its constitutive hexagons is minimal over all $k$-partitions.

Remark 4.17. As shown in Section 4.3, this is wrong for triangles and squares (see Figs. 19 and 20).

We explore this question by analyzing if weaker consequences of this conjecture are true. For example, Corollary 2.11 can be rephrased as follows.

Proposition 4.18. If the 'canonical' $k$-partition by hexagons is minimal, then for any extracted open set, the $k$-th eigenvalue of the Dirichlet Laplacian should be $\lambda_{1}\left(H e x a_{1}\right)$.

The partition of $\Omega \in \mathcal{H}^{(k)}$ by its hexagons is in general not bipartite but, as we have described previously, we can make it bipartite by considering $\Omega \backslash \cup_{j \in \sigma} \sigma_{j}$ where $\sigma_{j}$ is one side of the constitutive hexagons. Of course, this procedure is not unique so we can associate to $\Omega$ a family of such 'cut' open sets with cracks. Note that when the cut set is not connected any more, one can reinterpret the result as the direct sum of two independent spectral problems.

\subsubsection{3-Hexagons}

- 3-Hexagons without internal Dirichlet conditions, denoted by $H_{0}^{3}$ :

Numerical eigenpairs are given in Figure 21 and we deduce

$$
\begin{array}{ll}
\mathfrak{L}_{1}\left(H_{0}^{3}\right)=\lambda_{1}\left(H_{0}^{3}\right), & \mathfrak{L}_{2}\left(H_{0}^{3}\right)=\lambda_{2}\left(H_{0}^{3}\right)=\lambda_{3}\left(H_{0}^{3}\right), \\
\mathfrak{L}_{3}\left(H_{0}^{3}\right)<\lambda_{4}\left(H_{0}^{3}\right), & \mathfrak{L}_{4}\left(H_{0}^{3}\right)=\lambda_{4}\left(H_{0}^{3}\right) .
\end{array}
$$

- Cracked 3-hexagons $H_{1}^{3}$, see Figure 22:

The domain $H_{1}^{3}$ is obtained from $H_{0}^{3}$ by removing the interior horizontal side of the constitutive hexagons. It is clear that

$$
\mathfrak{L}_{3}\left(H_{0}^{3}\right) \leq \mathfrak{L}_{3}\left(H_{1}^{3}\right)=\lambda_{3}\left(H_{1}^{3}\right)=\lambda_{1}\left(\operatorname{Hexa}_{1}\right) .
$$

\subsubsection{7-Hexagons}

Let $H_{0}^{7}$ be a ring of 6 patterns $H e x a_{1}$ to which we add the middle pattern $H e x a_{1}$ (see Fig. 23). We construct new domains by removing some side of the constitutive hexagons to make the domain bipartite. After a possible symmetry, we can construct exactly 12 domains denoted by $H_{k}^{7}, k=1, \ldots, 12$ and drawn in Figure 24 .

We check that for any cracked 7-hexagons

$$
\mathfrak{L}_{7}\left(H_{0}^{7}\right) \leq \mathfrak{L}_{7}\left(H_{k}^{7}\right)=\lambda_{7}\left(H_{k}^{7}\right)=\lambda_{1}\left(H_{e x a}\right), \quad \text { for } k=1, \ldots, 12 .
$$

Numerical eigenvalues are given in Table 3 for any domain $H_{k}^{7}, k=0, \ldots, 12$. Figure 25 gives the first seven eigenfunctions of $H_{2}^{7}$. More simulations are available on [4]. Numerical computations confirm Conjecture 4.16. For $k=4,5,7,9,10$, the domain $H_{k}^{7}$ is not connected. Consequently, we notice that $\lambda_{1}\left(H e x a_{1}\right)$ arises before rank 7 .

We emphasize that this does not work in the same way as for the triangle, because we do have not selfsimilarity.

\subsection{Conclusion}

The presented simulations confirms the weaker form of Conjecture 4.16 (see Prop. 4.18) for $k=3$ and 7 . The same conclusion holds for the case $k=4$ which is fully analyzed in [4]. Some computations on a double ring of 18 patterns $H e x a 1_{1}$ to which we add the middle pattern $H e x a_{1}$ are also available. 

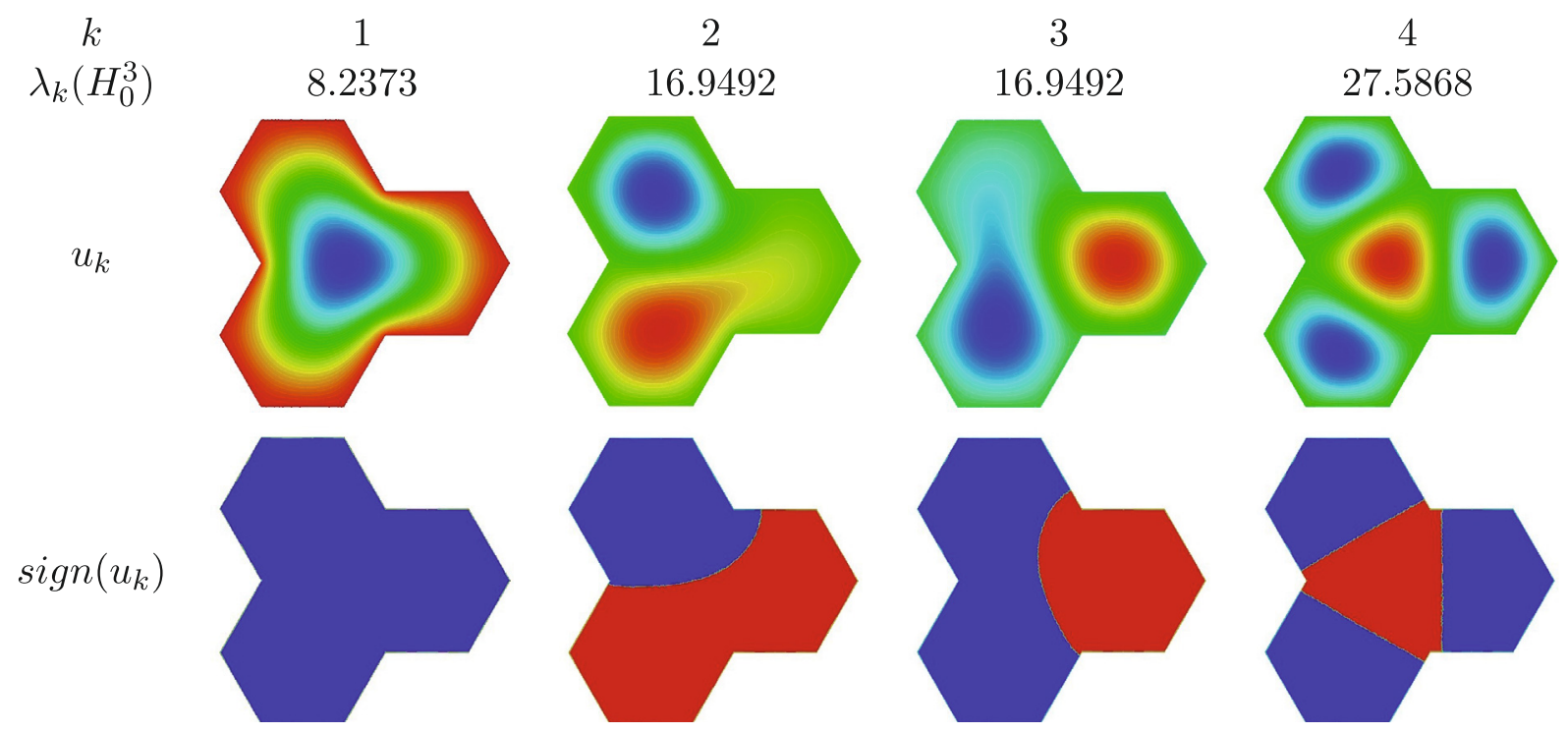

Figure 21. First four eigenmodes on 3-hexagons $H_{0}^{3}$.

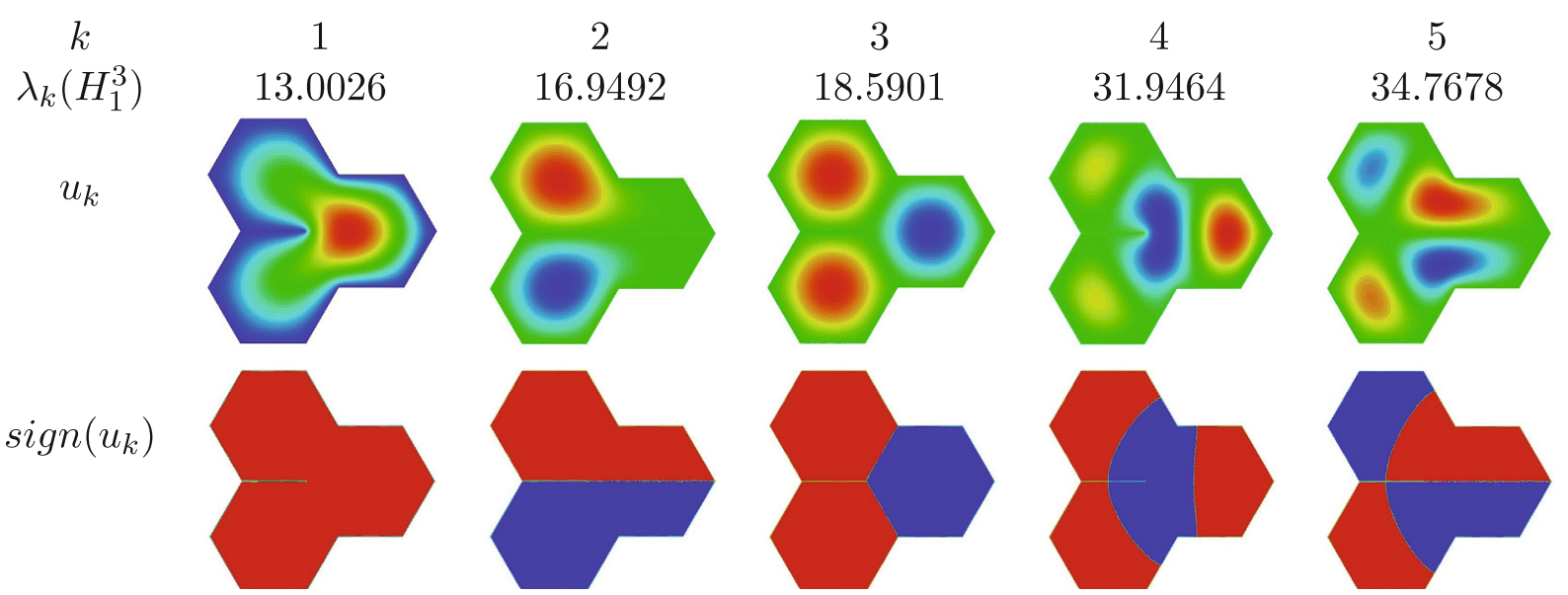

FiguRE 22. First five eigenmodes on cracked 3-hexagons $H_{1}^{3}$.

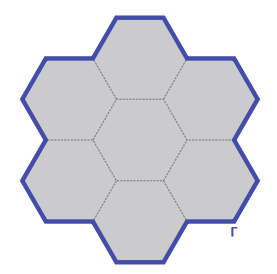

Figure 23. Domain with 7-hexagons $H_{0}^{7}$. 

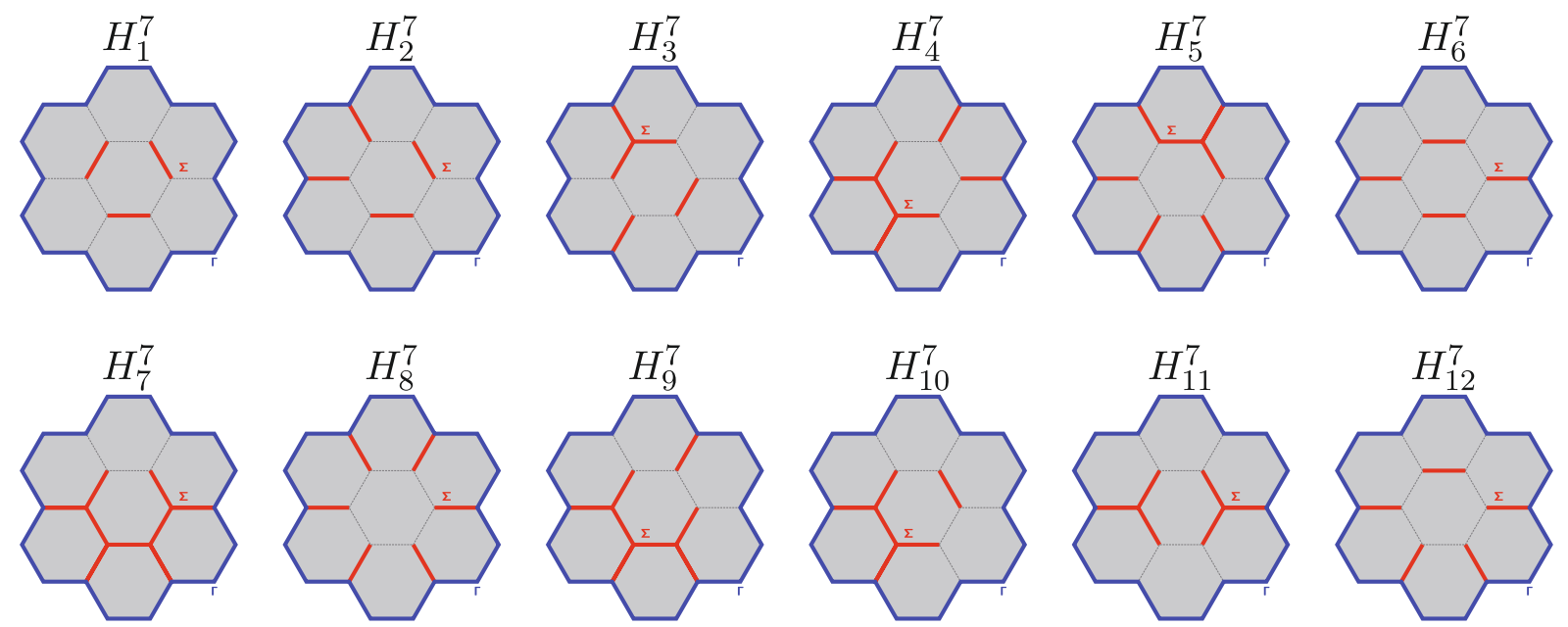

FigurE 24. All the bipartite configurations from 7-hexagons.

TABLE 3. First seven smallest eigenvalues for every configurations of 7-hexagons.

\begin{tabular}{|c|c|c|c|c|c|c|c|}
\hline$k$ & 1 & 2 & 3 & 4 & 5 & 6 & 7 \\
\hline$\lambda_{k}\left(H_{0}^{7}\right)$ & 2.9369 & 7.3544 & 7.3544 & 12.8445 & 12.8445 & 14.8399 & 17.2250 \\
$\lambda_{k}\left(H_{1}^{7}\right)$ & 10.3618 & 12.1236 & 12.1243 & 15.3192 & 15.7805 & 15.7807 & $\mathbf{1 8 . 5 9 0 1}$ \\
$\lambda_{k}\left(H_{2}^{7}\right)$ & 10.0981 & 12.4465 & 13.1570 & 14.6843 & 16.4286 & 17.1945 & $\mathbf{1 8 . 5 9 0 1}$ \\
$\lambda_{k}\left(H_{3}^{7}\right)$ & 10.7378 & 12.4433 & 13.6945 & 15.4251 & 16.7543 & 17.9174 & $\mathbf{1 8 . 5 9 0 1}$ \\
$\lambda_{k}\left(H_{4}^{7}\right)$ & 10.9022 & 13.4143 & 14.9745 & 17.5542 & 17.8478 & $\mathbf{1 8 . 5 9 0 1}$ & $\mathbf{1 8 . 5 9 0 1}$ \\
$\lambda_{k}\left(H_{5}^{7}\right)$ & 10.3645 & 13.7750 & 16.3271 & 17.0038 & 17.7148 & $\mathbf{1 8 . 5 9 0 1}$ & $\mathbf{1 8 . 5 9 0 1}$ \\
$\lambda_{k}\left(H_{6}^{7}\right)$ & 10.2306 & 12.9114 & 13.4639 & 14.5699 & 14.7908 & 17.6557 & $\mathbf{1 8 . 5 9 0 1}$ \\
$\lambda_{k}\left(H_{7}^{7}\right)$ & 11.5774 & 16.4434 & 17.0532 & $\mathbf{1 8 . 5 9 0 1}$ & $\mathbf{1 8 . 5 9 0 1}$ & $\mathbf{1 8 . 5 9 0 1}$ & $\mathbf{1 8 . 5 9 0 1}$ \\
$\lambda_{k}\left(H_{8}^{7}\right)$ & 8.7087 & 15.4547 & 15.4555 & 16.9274 & 16.9276 & 17.2250 & $\mathbf{1 8 . 5 9 0 1}$ \\
$\lambda_{k}\left(H_{9}^{7}\right)$ & 11.6285 & 13.7275 & 16.2064 & 17.9874 & $\mathbf{1 8 . 5 9 0 1}$ & $\mathbf{1 8 . 5 9 0 1}$ & $\mathbf{1 8 . 5 9 0 1}$ \\
$\lambda_{k}\left(H_{10}^{7}\right)$ & 10.9273 & 12.2217 & 15.4757 & 16.3846 & 17.2592 & $\mathbf{1 8 . 5 9 0 1}$ & $\mathbf{1 8 . 5 9 0 1}$ \\
$\lambda_{k}\left(H_{11}^{7}\right)$ & 11.2150 & 11.9100 & 16.3892 & 16.4434 & 16.4437 & 18.0900 & $\mathbf{1 8 . 5 9 0 1}$ \\
$\lambda_{k}\left(H_{12}^{7}\right)$ & 9.4695 & 13.2805 & 13.8475 & 16.3596 & 16.8214 & 17.3567 & $\mathbf{1 8 . 5 9 0 1}$ \\
\hline
\end{tabular}

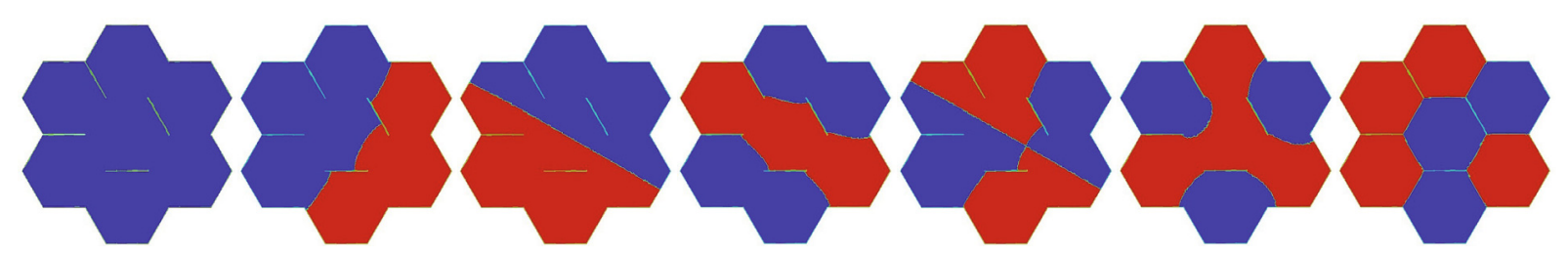

FiguRE 25. Nodal sets of the first seven eigenfunctions on $H_{2}^{7}$. 
A similar conjecture in favor of the hexagonal geometry appears in [7] for minimizing asymptotically the sum $\sum_{i=1}^{k} \lambda_{1}\left(D_{i}\right) / k^{2}$.

Acknowledgements. The authors would like to thank A. El Soufi, T. Hoffmann-Ostenhof, S. Terracini, M. Van den Berg and G. Verzini for many enlightening and stimulating discussions. The second author was partially supported by the ESF-programme SPECT. We also thank the referees for their useful comments.

\section{REFERENCES}

[1] G. Alessandrini, Nodal lines of eigenfunctions of the fixed membrane problem in general convex domains. Comment. Math. Helv. 69 (1994) 142-154.

[2] P. Bérard, Inégalités isopérimétriques et applications : domaines nodaux des fonctions propres. Exposé XI, Séminaire Goulaouic-Meyer-Schwartz (1982).

[3] L. Bers, Local behavior of solutions of general linear elliptic equations. Commun. Pure Appl. Math. 8 (1955) 473-496.

[4] V. Bonnaillie-Noël and G. Vial, Computations for nodal domains and spectral minimal partitions. http://w3.bretagne. ens-cachan.fr/math/simulations/MinimalPartitions (2007).

[5] D. Bucur, G. Buttazzo and A. Henrot, Existence results for some optimal partition problems. Adv. Math. Sci. Appl. 8 (1998) $571-579$.

[6] D. Bucur, B. Bourdin and E. Oudet, Numerical study of an optimal partitioning problem related to eigenvalues. (In preparation).

[7] L.A. Caffarelli and F.H. Lin, An optimal partition problem for eigenvalues. J. Sci. Comput. 31 (2007) 5-18.

[8] M. Conti, S. Terracini and G. Verzini, An optimal partition problem related to nonlinear eigenvalues. J. Funct. Anal. 198 (2003) $160-196$.

[9] M. Conti, S. Terracini and G. Verzini, A variational problem for the spatial segregation of reaction-diffusion systems. Indiana Univ. Math. J. 54 (2005) 779-815.

[10] M. Conti, S. Terracini and G. Verzini, On a class of optimal partition problems related to the Fucik spectrum and to the monotonicity formula. Calc. Var. 22 (2005) 45-72.

[11] O. Cybulski, V. Babin and R. Hołyst, Minimization of the Renyi entropy production in the space-partitioning process. Phys. Rev. E 71 (2005) 46130.

[12] B. Helffer, Domaines nodaux et partitions spectrales minimales (d'après B. Helffer, T. Hoffmann-Ostenhof et S. Terracini). Séminaire EDP de l'École Polytechnique (Déc. 2006).

[13] B. Helffer, On nodal domains and minimal spectral partitions. Conference in Montreal (April 2008).

[14] B. Helffer and T. Hoffmann-Ostenhof, Converse spectral problems for nodal domains. Mosc. Math. J. 7 (2007) 67-84.

[15] B. Helffer and T. Hoffmann-Ostenhof, On minimal partitions for the disk and the annulus. Provisory notes in February 2007.

[16] B. Helffer, T. Hoffmann-Ostenhof and S. Terracini, Nodal domains and spectral minimal partitions. Ann. Inst. H. Poincaré Anal. Non Linéaire (2007) doi:10.1016/j.anihpc.2007.07.004.

[17] D. Jakobson, M. Levitin, N. Nadirashvili and I. Polterovic, Spectral problems with mixed Dirichlet-Neumann boundary conditions: isospectrality and beyond. J. Comput. Appl. Math. 194 (2006) 141-155.

[18] N. Landais, Problèmes de régularité en optimisation de forme. Ph.D. Thesis, ENS Cachan Bretagne, France (2007).

[19] M. Levitin, L. Parnovski and I. Polterovich, Isospectral domains with mixed boundary conditions. J. Phys. A 39 (2006) 2073-2082.

[20] D. Martin, The finite element library Mélina. http://perso.univ-rennes1.fr/daniel.martin/melina (2006).

[21] A. Melas, On the nodal line of the second eigenfunction of the Laplacian on $\mathbb{R}^{2}$. J. Differential Geom. 35 (1992) $255-263$.

[22] A. Pleijel, Remarks on Courant's nodal theorem. Comm. Pure. Appl. Math 9 (1956) 543-550.

[23] G. Pólya, On the eigenvalues of vibrating membranes. Proc. London Mah. Soc. 3 (1961) 419-433. 